\title{
Autoantibodies against NMDA receptor 1 modify rather than cause encephalitis
}

\author{
Justus B. H. Wilke (D ${ }^{1}$, Martin Hindermann ${ }^{1}$, Stefan A. Berghoff ${ }^{2}$, Svenja Zihsler ${ }^{1}$, Sahab Arinrad', Anja Ronnenberg ${ }^{1}$, \\ Nadine Barnkothe ${ }^{1}$, Agnes A. Steixner-Kumar (iD) ${ }^{1}$, Stefan Röglin ${ }^{1}$, Winfried Stöcker ${ }^{3}$, Michael Hollmann ${ }^{4}$, Klaus-Armin Nave (iD ${ }^{2}$, \\ Fred Lühder ${ }^{5}$ and Hannelore Ehrenreich (iD ${ }^{1 凶}$
}

(c) The Author(s) 2021

The etiology and pathogenesis of "anti-N-methyl-D-aspartate-receptor (NMDAR) encephalitis" and the role of autoantibodies (AB) in this condition are still obscure. While NMDAR1-AB exert NMDAR-antagonistic properties by receptor internalization, no firm evidence exists to date that NMDAR1-AB by themselves induce brain inflammation/encephalitis. NMDAR1-AB of all immunoglobulin classes are highly frequent across mammals with multiple possible inducers and boosters. We hypothesized that "NMDAR encephalitis" results from any primary brain inflammation coinciding with the presence of NMDAR1-AB, which may shape the encephalitis phenotype. Thus, we tested whether following immunization with a "cocktail" of 4 NMDAR1 peptides, induction of a spatially and temporally defined sterile encephalitis by diphtheria toxin-mediated ablation of pyramidal neurons ("DTA" mice) would modify/ aggravate the ensuing phenotype. In addition, we tried to replicate a recent report claiming that immunizing just against the NMDAR1-N368/G369 region induced brain inflammation. Mice after DTA induction revealed a syndrome comprising hyperactivity, hippocampal learning/memory deficits, prefrontal cortical network dysfunction, lasting blood brain-barrier impairment, brain inflammation, mainly in hippocampal and cortical regions with pyramidal neuronal death, microgliosis, astrogliosis, modest immune cell infiltration, regional atrophy, and relative increases in parvalbumin-positive interneurons. The presence of NMDAR1-AB enhanced the hyperactivity (psychosis-like) phenotype, whereas all other readouts were identical to control-immunized DTA mice. Non-DTA mice with or without NMDAR1-AB were free of any encephalitic signs. Replication of the reported NMDAR1-N368/G369-immunizing protocol in two large independent cohorts of wild-type mice completely failed. To conclude, while NMDAR1-AB can contribute to the behavioral phenotype of an underlying encephalitis, induction of an encephalitis by NMDAR1-AB themselves remains to be proven.

Molecular Psychiatry (2021) 26:7746-7759; https://doi.org/10.1038/s41380-021-01238-3

\section{INTRODUCTION}

Since, the first report on a novel paraneoplastic autoimmune disease with autoantibodies against the NMDA receptor subunit NR1 (NMDAR1-AB $=$ GluN1-AB) appeared $>12$ years ago [1, 2], this condition attracted considerable attention both by clinicians and basic researchers worldwide, resulting in currently nearly 2000 entries in PubMed. Variably associated with psychosis, cognitive decline, extrapyramidal symptoms, or seizures, this "NMDAR encephalitis" was already early on described to be mediated by lower surface expression of neuronal NMDAR after exposure to NMDAR1-AB of the immunoglobulin $\mathrm{G}(\mathrm{lgG})$ class [3]. The resulting NMDAR antagonistic or "ketamine-like" effects, demonstrated also in various rodent models via passive transfer of NMDAR1-AB [4-13], were interpreted as potential mechanisms of action. Thus far, however, they have failed to explain the inflammatory or encephalitic component of this condition. Interestingly, comparison of NMDAR1-AB-positive and -negative human encephalitis cases did not reveal differences, except for few perhaps NMDAR-antagonistic (ketamine-like) symptoms [14].

Even sophisticated preclinical approaches raised further questions rather than providing answers. For example, immunization of mice with liposome-embedded tetrameric NMDAR was stated to induce NMDAR-AB and fulminant encephalitis. Highly variable behavioral changes in this model included hyperactivity, immobility, circling, seizures, hunched back/lethargy, and increased mortality, including neuroinflammation, and immune cell infiltration [15], all nonspecific features of any severe encephalitis. Adequate "negative" controls like the use of other liposomeembedded holoreceptors or of control proteins of similar size would have been required to link NMDAR-AB causally with the occurrence of an encephalitis. To validate that "disease induction depends on conformationally restricted epitopes" [15], infusion of purified NMDAR-AB should have been used to clarify whether they act encephalitogenic on their own [16].

During performance of the present work, a report claimed that immunizing mice against the NMDAR1-N368/G369 region alone at high dose induced encephalitis-like behavioral and morphological impairments, including blood brain-barrier (BBB) breakdown [17]. We thus decided to follow this initially exciting report's protocol, hoping to answer several important questions related to our own research. However, using two large independent cohorts of wild-

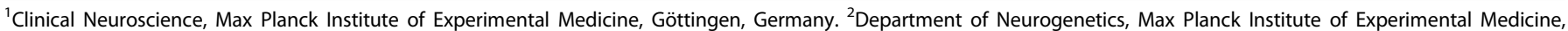

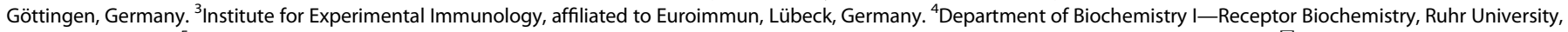

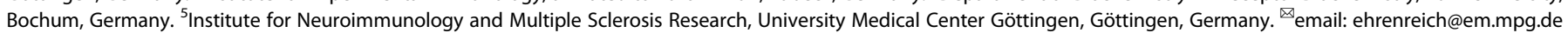


type mice, we were unable to reproduce any of the described findings. This replication failure is now also included in the present paper.

To summarize, despite all efforts, etiology and pathogenesis of the "NMDAR encephalitis" are still as uncertain as the role of NMDAR1-AB in this condition. We note that multiple braindirected $A B$ apart from NMDAR1-AB have been reported in serum of healthy humans and of various other mammalian species, likely belonging to the normal pre-existing "autoimmune repertoire." These $A B$ do have potential functionality and also potential pathogenicity [18-22]. In fact, functional serum NMDAR1-AB of all immunoglobulin classes are a highly frequent finding across mammals, with multiple possible inducers or boosters identified thus far, ranging from genetic predisposition, various viral infections (e.g., influenza, herpes), oncological conditions, and brain lesions to chronic life stress [1, 6, 23-26]. However, no solid proof exists to date that NMDAR1-AB by themselves can induce an encephalitic process.

The present study addresses the hypothesis that "NMDAR encephalitis" may result from a primary brain inflammation coinciding with the presence of NMDAR1-AB, which ultimately shape the encephalitis phenotype. Therefore, a spatially and temporally defined" sterile encephalitis was induced in young female NexCreERT2xRosa26-eGFP-DTA (= "DTA") mice after immunization with a cocktail of 4 NMDAR1 peptides, also including a peptide covering the NMDAR1-N368/G369 region. Females were chosen to account for the $\sim 4: 1$ female/male ratio observed in human "NMDAR encephalitis" [27]. As shown in vivo earlier, this cocktail induces functionally highly active NMDAR1-AB, leading to psychosis-like symptoms in mice with compromised BBB but no brain inflammation [28]. Here, we report that NMDAR1-AB can contribute to the behavioral abnormalities of an underlying gray-matter encephalitis, but that the multifaceted encephalitic phenotype itself, involving pyramidal neurons and their NMDAR, is nearly identical between NMDAR1-AB carriers and noncarriers.

\section{MATERIALS AND METHODS \\ Mice}

Mouse experiments (all C57BL/6 background) were approved by the Local Animal Care and Use Committee (LAVES, Niedersächsisches Landesamt für Verbraucherschutz und Lebensmittelsicherheit, Oldenburg, Germany) in accordance with the German animal protection law. Sample sizes were based on previous experience under consideration of the RRR-principle and technical limitations (i.e., maximum of 16 animals per IntelliCage). All experiments were performed by investigators unaware of group assignment (fully-blinded). Mice were separated by genotype and treatment to avoid inclusion effects [29] or aggressive behavior against potentially affected animals. Unless otherwise stated, mice were maintained in temperature- and humidity-controlled environments in $12 \mathrm{~h}$ light/dark cycle (light on at 7am) with wood-chip bedding, nesting material (Sizzle Nest, Datesand, Bredbury, UK) and, ad libitum food and water.

DTA cohort: Mice with the tamoxifen-inducible gray-matter inflammation were generated by crossing homozygous Neurod6 $6^{\text {tm2.1(cre/ERT2)Kan }}$

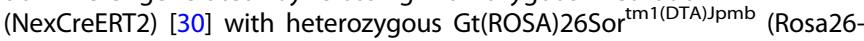
eGFP-DTA) [31], resulting in double-heterozygous (DTA) mice and heterozygous NexCreERT2 littermate (control) mice lacking the DTA allele. Detailed genotyping protocols are available upon request. Experiments involving DTA mice were performed on females to account for the $~ 4: 1$ female/male ratio observed in human "NMDAR encephalitis" patients [27]. Female transgenic mice were weaned at postnatal day 21 into type IV cages $(55 \times 38.5 \times 20.5 \mathrm{~cm}$, Tecniplast, Hohenpeißenberg, Germany) in groups of 16 .

Replication cohorts comprised male C57BL/ $6 \mathrm{~J}$ wildtype mice immunized at 8-9 weeks of age [17]. Wildtype mice were obtained from Janvier (Le Genest-Saint-Isle, France), transported to our behavior unit at 3 weeks of age, and housed in type II cages $(36.5 \times 20.7 \times 14 \mathrm{~cm}$, Tecniplast $)$ in groups of 3-5.

\section{Treatments}

Immunization of the DTA cohort was conducted as previously described [28], except that immunizations were performed on postnatal day 30 . Mice were immunized with a cocktail of $4 \mathrm{GluN1}$ extracellular peptides (GluN1 $1_{35-53}$ GluN1 $1_{361-376,}$ GluN1 $1_{385-399}$, and GluN1 $1_{660-811}$ coupled to keyhole limpet hemocyanin; Synaptic Systems, Göttingen, Germany) and/or chicken ovalbumin ("OVA", A5503, Sigma-Aldrich, Darmstadt, Germany) emulsified in an equal volume of complete Freund's adjuvant (CFA) containing $1 \mathrm{mg} / \mathrm{mL}$ heat-killed Mycobacterium tuberculosis H37 Ra (\#231141, Difco, BD, Heidelberg, Germany) in incomplete Freund's adjuvant. GluN1 peptide cocktail $(50 \mu \mathrm{g})$ and/or ovalbumin $(20 \mu \mathrm{g})$ were injected subcutaneously at the tail base.

Immunization of the replication cohorts followed the protocol of Wagnon et al. [17]. Male C57B/6 J mice were immunized at 8-9 weeks of age with either GluN1 $1_{168-187}$ (control peptide), GluN1 $1_{359-378}$ (active peptide) or for our additional comparison with ovalbumin, each emulsified in an equal volume of CFA (as described above). Antigens ( $200 \mu \mathrm{g}$ ) were equally distributed over 4 subcutaneous injections, 2 at shoulders and 2 at hind limbs. In addition, mice received 2 intraperitoneal injections of $200 \mathrm{ng}$ of Bordetella pertussis toxin (\#180, List Biological Laboratories) in PBS, immediately after and $48 \mathrm{~h}$ after immunization.

Tamoxifen induction: Tamoxifen (CAS\#10540-29-1, T5648, Sigma) was dissolved in corn oil (C8267, Sigma) on injection days at $10 \mathrm{mg} / \mathrm{mL}$. Mice received 2 intraperitoneal injections of $100 \mathrm{mg}$ of tamoxifen $/ \mathrm{kg}$ body weight on 2 consecutive days.

Transponder placement: For the experimenter-independent phenotyping of mice in the IntelliCage apparatus (TSE Systems, Bad Homburg, Germany) ISO standard transponders $(8.5 \mathrm{~mm}$ length, $1.2 \mathrm{~mm}$ diameter, PM162-8) were implanted below the skin of the neck after intraperitoneal injection of $24 \mu \mathrm{L}$ of $1.36 \%$ 2,2,2,-tribromoethanol (T48402, Sigma) in $\mathrm{ddH}_{2} \mathrm{O} / \mathrm{g}$ body weight (Avertin). One week after implantation, mice were placed into IntelliCages.

Blood sampling: Intermediate blood samples $(100 \mu \mathrm{L})$ were collected from the retro-orbital sinus. Terminal blood $(500 \mu \mathrm{L})$ was sampled by cardiac puncture before transcardial perfusion. EDTA-plasma aliquots were stored at $-80^{\circ} \mathrm{C}$.

\section{Behavioral phenotyping}

Experiments of the DTA cohort were performed in the following order: LABORAS (baseline-prior to tamoxifen induction), bar test, hurdle test, IntelliCage-based phenotyping including pheromone preference, LABORAS, Morris water maze, hole board, prepulse inhibition (PPI), marble-burying test, and complex wheel running.

Behavioral testing of the replication cohort followed the design of Wagnon et al. [17]. Behavioral analyses comprised open-field activity, Ymaze working memory, elevated plus maze (anxiety), and forced-swim test (depression-like behavior). For the second replication cohort, we rearranged the test schedule to assess anxiety-like behavior in behaviorally naive mice, followed by testing in open field and Y-maze.

Except for home cage-based tests, all tests ran during the light phase. Behavioral testing was performed as previously described in detail $[17,29,32-38]$. Only home cage-based tests are briefly summarized below.

\section{LABORAS}

To characterize the spontaneous home cage behavior of mice prior to and after a tamoxifen-induced gray-matter inflammation, the LABORAS system (Metris B.V.) was employed [32-34, 37]. Briefly, mice habituated for 2 nights to the experimental room and single housing in clear polycarbonate cages (Makrolon type I, $22 \mathrm{~cm} \times 16 \mathrm{~cm} \times 14 \mathrm{~cm}$, Tecniplast) with wood-chip bedding, food, and water ad libitum. After two nights, cages were placed on a sensor platform (Carbon Fiber Plate $1000 \mathrm{~mm} \times 700 \mathrm{~mm} \times 700 \mathrm{~mm} \times$ $30 \mathrm{~mm}$, Metris B.V., Hoofddorp, Netherland) and the resulting electrical signals recorded throughout the dark phase $(12 \mathrm{~h})$ and classified into behavioral categories, i.e., eating, drinking, scratching, circling, climbing, immobility, locomotion, and grooming.

\section{IntelliCage-based phenotyping}

To assess a variety of cognitive measures with minimal experimenter intervention on a $24 / 7$ basis in a social home cage-based setting, 16 mice per group were placed in standard laboratory rodent cages $(55 \times 38.5 \times$ $20.5 \mathrm{~cm}$, Tecniplast) equipped with the IntelliCage apparatus (TSESystems) controlled by NewBehavior software (version 3.1.7), as we 
described in great detail previously [29]. Water was accessible in four triangular conditioning chambers $(15 \times 15 \times 21 \mathrm{~cm})$, located in cage corners. The conditioning chambers were equipped with temperature sensors and RFID antennas to identify entering mice. If mice entered a correct corner during the allocated time window, doors blocking access to water or sucrose bottles (dependent on test setting) could be opened via nosepokes sensed by light barriers. Lickometers registered licks on bottles.

The experimental setup comprised measurement of place learning (day 1 ), reversal learning (day 2), sucrose preference (day 3 ), acquisition and recollection of a place time-reward/episodic-like memory (days 4 and 5), and behavioral extinction of operant responses (days 6 and 7). General activity and day/night pattern were assessed by recording number and timing of visits to operant chambers (corner visits). On days $1-2$, each mouse had access to water at a single corner (4 mice/corner), during days $3-5$ to a $2 \%$ sucrose solution on one corner and water at the opposite site (8 mice/corner). On days 6-7, mice had access to only water at previously rewarded corners. In-between test sessions, corners were re allocated. The efficiency of place and reversal learning was assessed for $24 \mathrm{~h}$ each by calculating the percentage of place errors (visits to blocked corner/total corner visits $\left.{ }^{*} 100 \%\right)$. Sucrose preference means the percentage of licks at sucrose bottle/total licks within $24 \mathrm{~h}$. To evaluate episodic-like memory, access to water and sucrose solution was restricted to the first $2 \mathrm{~h}$ after dark-phase onset. Within this $2 \mathrm{~h}$ period, preference to sucrose corner was calculated as percentage of visits to sucrose corner/total corner visits.

Two weeks after this IntelliCage testing, mice returned to IntelliCages to evaluate pheromone preference using two social boxes, supplemented with fresh wood-chip bedding, connected to the left and right side of the IntelliCage via two plastic tubes, equipped with two ring RFID antennas to track individual mouse. The time each mouse spent in the IntelliCage, the neutral or the target social box was recorded along with the number of social box visits. After habituation of $1 \mathrm{~h}$, social boxes were replaced by novel cages filled with either fresh wood-chip bedding (neutral site) or bedding from male $\mathrm{C} 3 \mathrm{H}$ mice (target site) and mice again had $1 \mathrm{~h}$ to explore the social boxes. In addition to time spent on the target site, the time spent exploring either social box was evaluated as the readout for exploratory behavior.

\section{Complex wheel running}

To assess locomotor activity and motor-cognitive learning, a complex running-wheel-setup (CRW) was used. Mice were single-housed in type-III cages $(42 \times 26 \times 18 \mathrm{~cm}$, Tecniplast), equipped with CRW (TSE-Systems). CRW are characterized by randomized omitted bars [39-41]. Mice were habituated to the experimental room and CRW for $2 \mathrm{~h}$ prior to the dark phase. After dark-phase onset, voluntary running was automatically tracked for $4 \mathrm{~h}$ via Phenomaster software (TSE-Systems) and the total running distance per mouse calculated.

\section{Antibody determinations}

ELISA: Immunizations were confirmed by antigen ELISA, at 21-27 days after immunization in the DTA cohort and at 17-18 days in replication cohorts. ELISA was performed as described [28]. ELISA plates (96-well Fbottom Immuno MediSorp, Nunc) were coated overnight at $4^{\circ} \mathrm{C}$ with either $0.2 \mu \mathrm{g}$ ovalbumin, $0.5 \mu \mathrm{g}$ GluN1-antigen cocktail, $1 \mu \mathrm{g}$ GluN1 ${ }_{168-187}$ or $1 \mu \mathrm{g} \mathrm{GluN1} 1_{359-378}$ in $50 \mu \mathrm{L}$ PBS per well. After blocking with $5 \%$ bovine serum albumin (BSA, \#8076.3, Roth) in PBS, mouse plasma (1:1000 dilution with $5 \%$ BSA/PBS $50 \mu \mathrm{l} /$ well) was added for $2 \mathrm{~h}$ at RT. Antigen-specific lgG antibodies were detected using HRP-coupled anti-mouse-lgG-specific antibodies (1:10,000, A9917, Sigma) and 3,3',5,5'-tetramethylbenzidine substrate (\#555214, BD OptEIA, BD). Absorbance at $450 \mathrm{~nm}$ was measured and corrected for values at $620 \mathrm{~nm}$ by microplate reader (Tecan-Trading AG, Männedorf, Switzerland). The threshold for a positive classification was set as three standard deviations above the mean of control samples.

Cell-based assay (CBA) for NMDAR1-AB detection: To determine NMDAR1-AB IgG titers against full-length GluN1, a commercially available cell-based assay comprising Grin1-transfected and control-transfected HEK293 cells (FB 112d-1010-51, EUROIMMUN, Lübeck, Germany) was used according to the manufacturer's instructions with minor modifications. The anti-human secondary antibody solution was replaced by Alexa Fluor 488labeled anti-mouse IgG (1:1000, A21202, Thermo Fisher Scientific, Darmstadt, Germany) in $0.2 \%$ Tween20/PBS. Titers were determined starting at 1:100 dilutions with subsequent testing of positive samples at 1:1000 and 1:10,000 and independently evaluated by three investigators. For colocalization experiments, diluted plasma samples were spiked with a non overlapping rabbit IgG anti-GluN1 antibody directed against the Cterminal domain (1:1000, G8913, Sigma) and an Alexa Fluor 647-labeled anti-rabbit IgG antibody (1:1000, A31573, Thermo) was added to the secondary antibody solution. Images were acquired on a confocal microscope (LSM880, Zeiss, Oberkochen, Germany).

\section{Measurements assessing blood-brain-barrier integrity}

$B B B$ integrity was evaluated as previously described $[28,42]$. Briefly, mice received intravenous injections of Evans blue $(50 \mu \mathrm{g} / \mathrm{g}$ body weight, E2129, Sigma) and sodium fluorescein $(200 \mu \mathrm{g} / \mathrm{g}$ body weight, F6377, Sigma). After $4 \mathrm{~h}$, mice were anesthetized and transcardially perfused. Brains were collected, frozen on dry ice, and lyophilized. Tracers were extracted from hemispheres with formamide and quantified in triplicates on a fluorescent microscope (Observer Z2, Zeiss). The concentrations of tracers were calculated using a standard curve and normalized to controls.

\section{Histology}

Mice were anesthetized with Avertin, transcardially perfused with Ringer (B. Braun, Melsungen, Germany) and subsequently $4 \%$ formaldehyde/PBS solution. Brains were collected, post fixed in $4 \%$ formaldehyde/PBS for $12 \mathrm{~h}$, dehydrated in 30\% sucrose/PBS for $48 \mathrm{~h}$, embedded in optimal cutting medium (Tissue-Tek, \#4583, Sakura, Umkirch, Germany) and frozen on dry ice. Frozen brains were cut into $30 \mu \mathrm{m}$ coronal sections on a cryostat (CM1950, Leica, Wetzlar, Germany), and stored at $-20^{\circ} \mathrm{C}$ in $25 \%$ ethylene glycol/25\% glycerol/PBS. Quantifications were performed on regularly spaced sections (every $300 \mu \mathrm{m}$ ) between Bregma coordinates -1.34 to $-2.24 \mathrm{~mm}$ and $4-6$ hippocampi from 2 to 3 sections were used per mouse. CA2/CA3 region is referred to as CA3 in text and figures.

Free-floating frozen sections were blocked and permeabilized for $1 \mathrm{~h}$ at RT with 5\% normal horse serum (NHS, 26050-088, Thermo) in 0.5\% Triton $\mathrm{X}-100 / \mathrm{PBS}$, incubated overnight at $4{ }^{\circ} \mathrm{C}$ with primary antibodies and subsequently stained with the corresponding fluorescently labeled secondary antibodies, for $2 \mathrm{~h}$ at RT. Nuclei were stained for $10 \mathrm{~min}$ at RT with $0.2 \mu \mathrm{g} / \mathrm{mL}$ 4',6-diamidino-2-phenylindole in PBS (DAPI, D9542, Sigma) and sections mounted on SuperFrost ${ }^{\oplus}$-Plus slides (J1800AMNZ, Thermo) with Aqua-Poly/Mount (\#18606, Polysciences, Warrington, PA, USA). The following primary antibodies were used: Mouse anti-GFAP (1:500, NCLGFAP-GA5, NovoCastra-Leica, Newcastle upon Tyne, UK), guinea pig antiS100b (1:750, \#287004, Synaptic Systems), rabbit anti-Iba1 (1:1000, \#01919,741, Wako, Neuss, Germany), rat anti-CD68 (1:500, MCA1957GA, BioRad, München, Germany), rat anti-CD45 (1:100, \#103101, BioLegend, Koblenz, Germany), and guinea pig anti-parvalbumin (1:1000, \#195004, Synaptic Systems). The corresponding secondary antibodies included Alexa Fluor 555 anti-rabbit (1:1000, A21428, Thermo), Alexa Fluor 555 anti-mouse (1:1000, A31570, Thermo), Alexa Fluor 647 anti-mouse (1:1000, A31571, Thermo), Alexa Fluor 633 anti-guinea pig (1:1000, A21105, Thermo), Alexa Fluor 647 anti-rat (1:1000, A21247, Thermo). For Fluorojade C-staining (AG325, Sigma) of dying neurons, sections were incubated in $0.06 \%$ potassium permanganate solution for $10 \mathrm{~min}$. Following a $1 \mathrm{~min}$ water rinse, tissue was transferred for $10 \mathrm{~min}$ to a $0.0001 \%$ solution of Fluorojade $\mathrm{C}$, dissolved in $0.1 \%$ acetic acid. Slides were rinsed with $\mathrm{ddH} 2 \mathrm{O}$ and dried at $60^{\circ} \mathrm{C}$. Slides were mounted with Aqua-Poly/Mount (\#18606, Polysciences). Overview images of whole-brain sections were obtained on Eclipse-TI 2 epifluorescence microscope (Nikon, Düsseldorf, Germany), equipped with $4 \times$ objective (4×/0.2 NA PLAN APO \#MRD00045, Nikon). For quantification, $1 \mu \mathrm{m}$-thick optical sections of hippocampi were acquired as tile scans on a confocal laser scanning microscope (LSM 880 , Zeiss), furnished with a $40 \times$ oil objective $(40 \times / 1.4$ NA PlanAPOCHROMAT, \#420762-9900, Zeiss). Image-acquisition parameters were kept constant within experiments. Quantifications and image processing were performed with FIJI-ImageJ software [43]. Iba1+ cells (mostly microglia), parvalbumin + cells (inhibitory neurons), and CD45+ lba1cells (leukocytes) were manually counted. GFAP+ area was quantified densitometrically upon uniform thresholding. Cell counts and GFAP + area were normalized to quantified areas. Data from 4 to 6 hippocampi/mouse was averaged.

\section{Flow cytometry}

Mice were anesthetized with Avertin and transcardially perfused with 40 $\mathrm{mL}$ of Ringer solution (B. Braun). Brains were stored on ice in $10 \%$ fetal bovine serum (FBS, \#10500-064, Thermo)/DMEM (\#41965, Thermo). Olfactory bulbs and brain stems were removed and brains meshed 


\section{A Schematic description of the construct}

Gt(ROSA)26Sortm1(DTA)Jpmb $\rightarrow$ 'DTA allele'

\begin{tabular}{|l||l|l|l|l|l|l|}
\hline eGFP & PGK-Neo tpA & $>$ & DTA & & bpA & \\
\hline
\end{tabular}

Tamoxifen-dependent Cre-mediated excision of eGFP-STOP cassette

\begin{tabular}{|l|l|l|l|l|}
\hline \hline & DTA & & bpA & \\
\hline
\end{tabular}

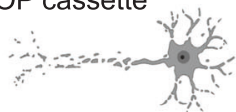

B Experimental validation of DTA model

NexCreERT2xRosa26-eGFP-DTA (='DTA')

Tamoxifen

(or corn oil control)

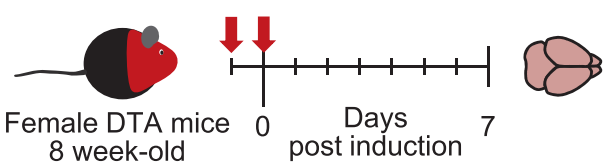

\section{Fluorojade C staining demonstrating neurodegeneration in DTA mice}

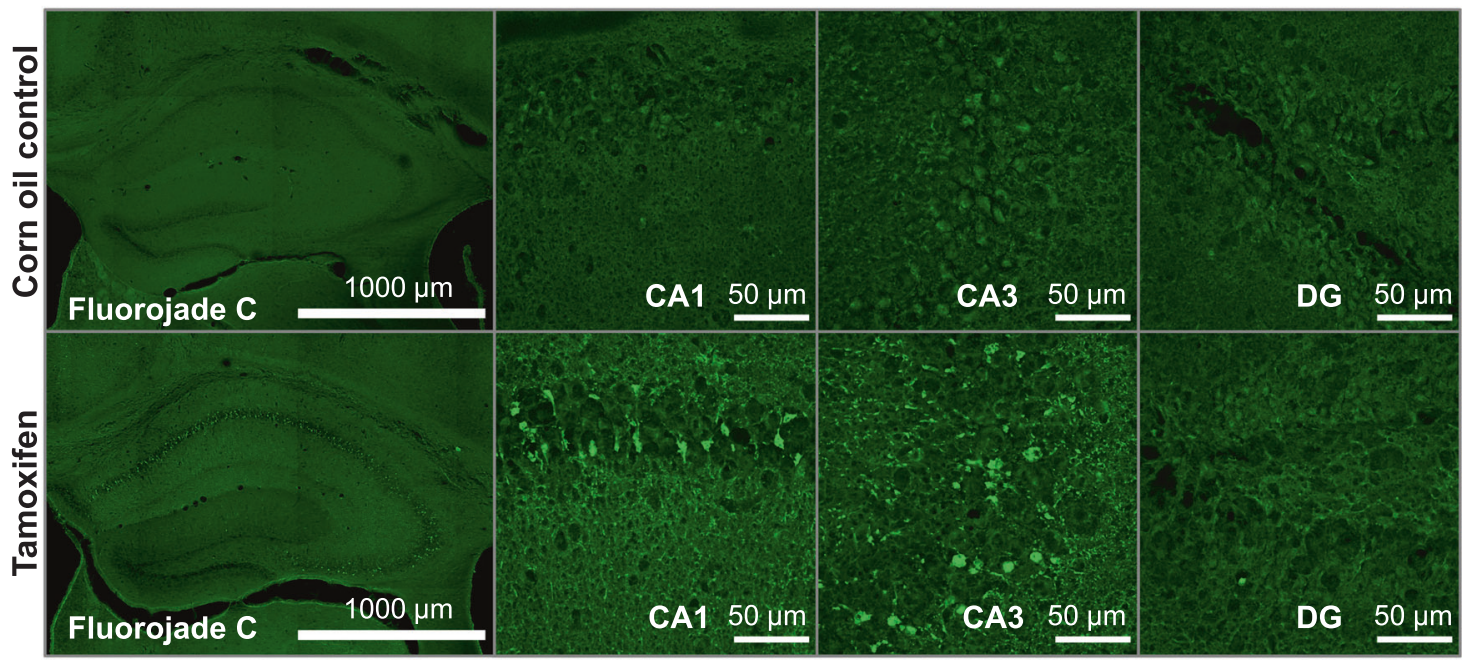

\section{Acute inflammatory response after tamoxifen-induced neuronal death in DTA mice}

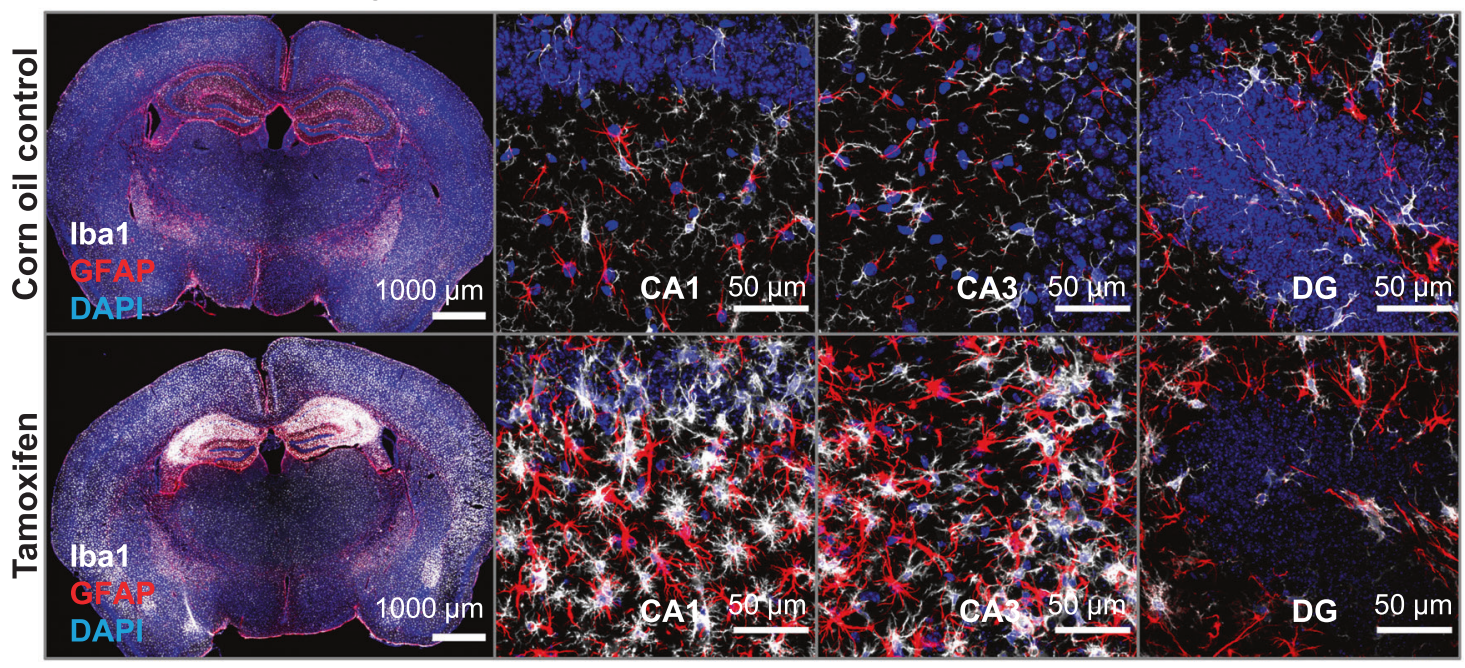

Fig. 1 Acute inflammatory response upon tamoxifen-induced Cre recombination in NexCreERT2xRosa26-eGFP-DTA (DTA) mice. A Schematic description of the Rosa26-eGFP-DTA allele. Upon tamoxifen-dependent Cre translocation, a loxP- (triangles) flanked eGFP-STOP cassette is excised, resulting in expression of diphtheria toxin chain A and ultimately cell death. B Experimental validation of the acute inflammatory response in DTA mice. Female DTA mice, 8-week old, received 2 intraperitoneal injections of either tamoxifen or solvent control (corn oil). C, D Brains were collected after 7 days and stained for neurodegeneration/cell death with Fluorojade C, as well as microglia (Iba1) and astrocytes (GFAP) as indicators of reactive gliosis. High-resolution images of CA1, CA3, and dentate gyrus (DG) regions were acquired as $10 \mu \mathrm{m}$ Z-stacks and are displayed as maximum-intensity projections.

through $70 \mu \mathrm{m}$ cell strainers. Cells were suspended in isotonic Percoll (17-0891-01, Sigma) to a final concentration of $30 \%$ and centrifuged to remove myelin. Cells were washed with FACS buffer ( $2 \%$ FBS, 10 mM EDTA in PBS) and filtered through $40 \mu \mathrm{m}$ cell strainers. Fc receptors were blocked for $10 \mathrm{~min}$ at $4^{\circ} \mathrm{C}$ with anti-mouse CD16/32 antibodies (1:100, 14-0161, Thermo). Cells were stained for $30 \mathrm{~min}$ at $4{ }^{\circ} \mathrm{C}$ with the following antibody mix: APC anti-CD45 (1:200, clone 104, BioLegend), PE anti-CD11b (1:200, clone M1/70, BioLegend), PECy5 anti-CD4 (1:1000, clone H129.19, BioLegend), PECy7 anti-CD8 (1:500, clone 53-6.7, Biolegend), APCCy7 anti-CD19 (1:200, clone 6D5, BioLegend), FITC anti-B220 (1:250, clone RA36B2, BioLegend), and PerCP-Cy5.5 anti-CD138 (1:200, clone 281-2, BioLegend). After staining, cells were washed and suspended in $400 \mu \mathrm{L}$ of FACS buffer and $100 \mu \mathrm{L}$ of APC quantification beads (\#340487, BD). Samples were measured on a FACSAria Sorp (BD) and CytoFLEX $S$ 


\section{A Experimental outline}

Experimental groups:

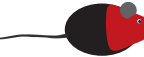

CTRL-OVA

DTA-OVA

DTA-GluN1

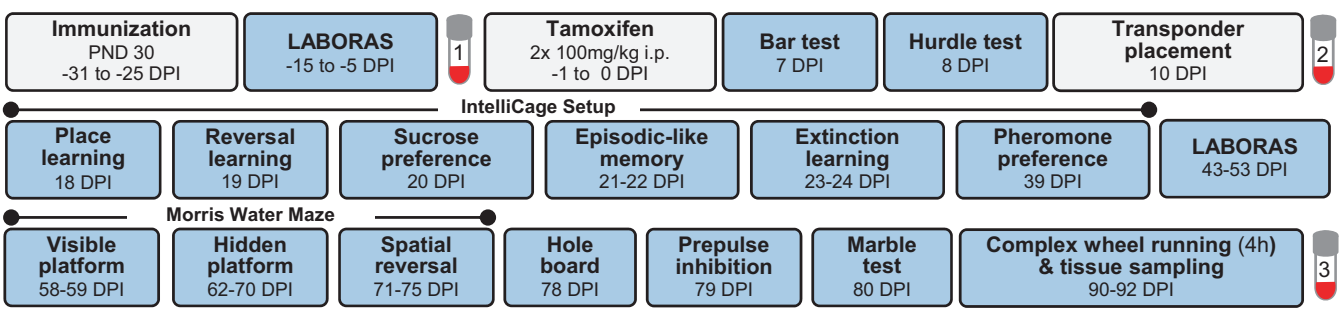

B GluN1 cocktail ELISA C GluN1 cell based assay

(Time point 1)
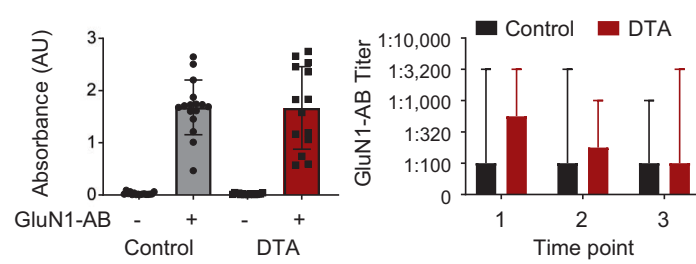

D Immunocytochemistry: GluN1 cell based assay

E LABORAS

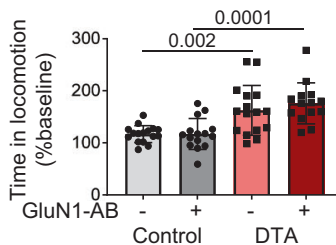

I MWM visible - day 1

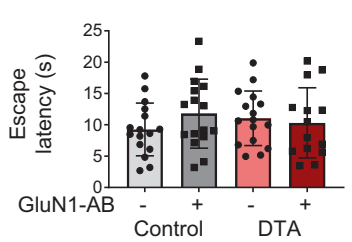

F IntelliCage Activity

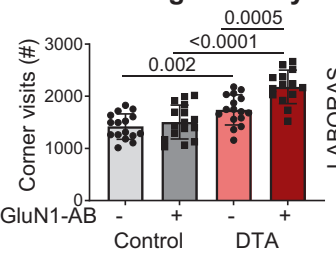

J MWM visible - day 2

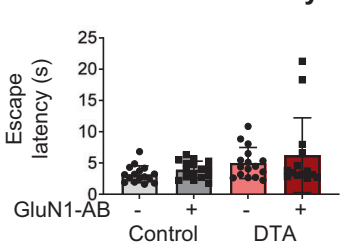

L MWM hidden platform

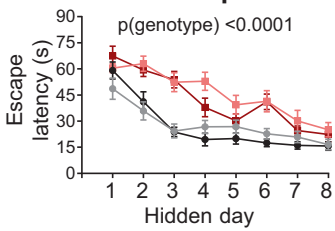

M

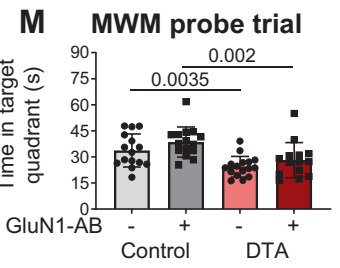

P Prepulse Inhibition

0.087

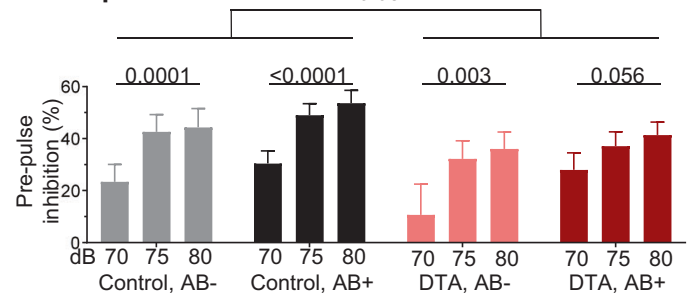

(Beckman Coulter, Krefeld, Germany). Cell numbers were corrected for the number of recorded APC beads. Leukocytes (CD45 high, $\left.C D 11 b^{\text {low }}\right)$ and microglia/macrophages (CD45 ${ }^{\mathrm{mid}}, \mathrm{CD} 11 \mathrm{~b}^{\text {high }}$ ) were quantified within single-cell gate determined by forward and side scatter. $\mathrm{CD}^{+}$and $\mathrm{CD} 8^{+}$ T-cells were quantified within leukocyte gate. $\mathrm{CD} 19^{+} \mathrm{B}$ cells and $\mathrm{CD} 138^{+}$ plasma cells were quantified in $\mathrm{CD}^{-} \mathrm{CD}^{-}$leukocyte gate.

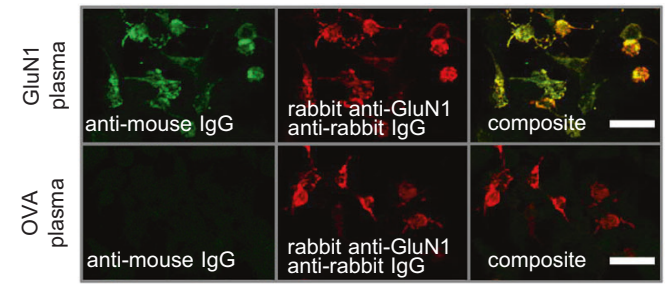

G Activity correlation $\mathrm{H}$ Complex wheel running
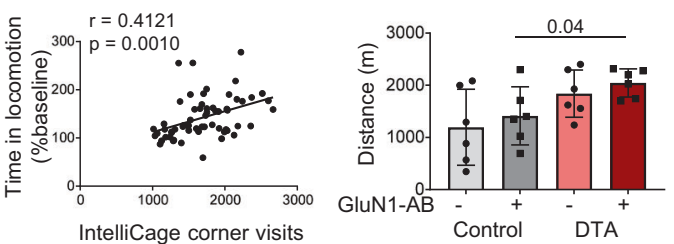

K MWM visible platform - task learning
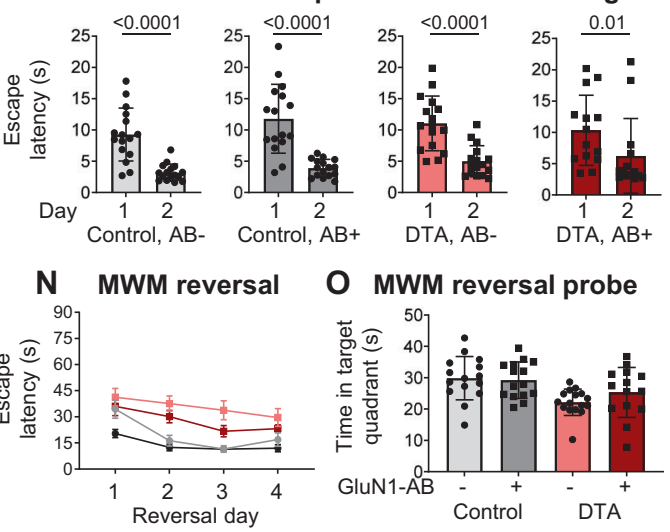

O MWM reversal probe
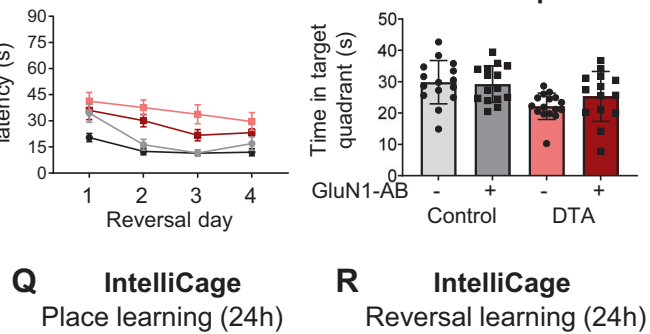

R IntelliCage Reversal learning (24h)
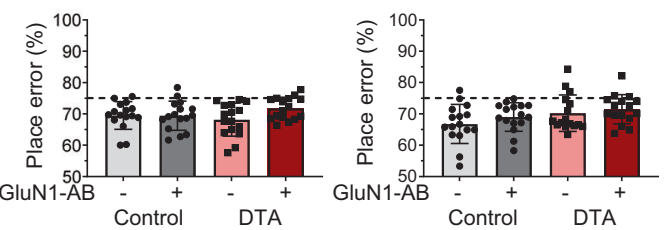

\section{Statistical analysis}

Statistical analyses were performed using Prism software (GraphPad Software) with the exception of the mixed ANOVA that was conducted on PPI data with R4.0.3 [44] using the rstatix package [45]. The results are presented as mean \pm standard deviations (SD), with few exceptions as indicated in the figure legends. Data normality was assessed using the 
Fig. 2 Pathophysiological relevance of NMDAR1-AB (=GluN1-AB) in the context of gray-matter inflammation. A Experimental outline indicating experimental groups, as well as order and time of behavioral tests (DPI = days post induction, i.e., after the last tamoxifen injection). B NMDAR1-AB validation by ELISA. C Cell-based (HEK293T) clinical standard assay for NMDAR1-AB (Euroimmun). D Immunocytochemical colocalization (CBA, Euroimmun) with a commercial rabbit GluN1-AB directed against the C-terminal domain. $\mathbf{E}$ Intra individual change of locomotor activity assessed in LABORAS at baseline and after tamoxifen induction. For each mouse, time in locomotion after tamoxifen induction was normalized to time in locomotion prior to tamoxifen application (baseline). F Activity (number of corner visits) over a 7-day IntelliCage session. G Pearson correlation between IntelliCage activity and intra individual changes of LABORAS locomotor activity. H Locomotor activity assessed by $4 \mathrm{~h}$ voluntary complex wheel running. I-O Cognitive testing in Morris water maze (MWM). I-K Visible platform task comprising 2 training days, demonstrating the ability for fast escape and simple task learning using within-maze cues. $\mathbf{L}$ Training of hidden platform task using extra-maze cues. DTA mice performed significantly worse than control mice (repeated-measures ANOVA, $p<$ $0.0001)$, whereas no effect of NMDAR1-AB was observed in either DTA $(p=0.3273)$ or control mice $(p=0.5972)$. M Evaluation of spatial memory in the probe trial. $\mathbf{N}$ Spatial reversal of the hidden platform. $\mathbf{O}$ Evaluation of cognitive flexibility and reversal learning in a second probe trial after spatial reversal training. $\mathbf{P}$ Prepulse inhibition of acoustic startle. Intra group comparisons performed using repeated-measure one-way ANOVA; inter group comparison between genotypes for 75 and $80 \mathrm{~dB}$ prepulses performed using mixed ANOVA. Q IntelliCage-based evaluation of place learning and $\mathbf{R}$ reversal learning within $24 \mathrm{~h}$ sessions shows similar performance across groups. Dashed lines indicate performance at chance level (75\%). Experiments were performed with 14-16 mice/group, except for CRW (H, $n=6$ mice/group). Data presented as mean $\pm S D$, except for repeated measure data $(\mathbf{L}, \mathbf{N}, \mathbf{P}$; mean $\pm S E M)$ and $A B$ titers $(\mathbf{C}$, median, range).

Shapiro-Wilk test with an alpha error of 0.05 . Dependent on data distribution, two-tailed unpaired Welch's-corrected $t$-tests or Mann-Whitney $U$-tests were used to compare groups of 2 . Similarly, repeatedmeasure ANOVA, one-way ANOVA or Kruskal-Wallis test were used to compare multiple groups. $P$ values $<0.05$ were considered statistically significant.

\section{RESULTS AND DISCUSSION}

Diphtheria toxin-mediated ablation of pyramidal neurons to mimic encephalitis affecting primarily gray matter

To mimic features of a viral encephalitis in a spatially and temporally defined sterile experimental approach, we employed young female NexCreERT2xRosa26-eGFP-DTA ( = "DTA") mice with an inducible transgene for diphtheria toxin-mediated ablation of pyramidal neurons, generating gray-matter inflammation $[30,31,46]$. Cell death can be controlled by dosing tamoxifen. Thus, in a series of dose-titrating pilot experiments, we selected a 2-day tamoxifen-injection design (Fig. 1A, B). After 7 days, we histologically confirmed acute neurodegeneration, pyramidal neuron loss, and a distinct inflammatory response comprising micro- and astrogliosis (Fig. 1C, D).

\section{Generation of primary brain inflammation coinciding with the} controlled presence of NMDAR1-AB following immunization We next addressed our hypothesis that "NMDAR encephalitis" may result from a primary brain inflammation concurring with the presence of NMDAR1-AB, which ultimately shape the encephalitis phenotype. Therefore, the above-described, defined sterile encephalitis was induced in young female DTA mice after active immunization with a cocktail of four peptides of extracellular NMDAR1/GluN1 domains, including a peptide covering the NMDAR1-N368/G369 region, versus ovalbumin as control immunization. This cocktail induces sufficient titers of highly functional NMDAR1-AB of the IgG class [28]. Active-immunization was performed at postnatal day 30, followed by an experimental scheme as detailed in Fig. 2A, comprising induction of encephalitis, extensive behavioral testing, blood sampling, and finally perfusion.

Immunization of mice against the 4 NMDAR1 peptides led to high circulating levels (even though somewhat variable between subjects) of specific $A B$, as shown by ELISA and cell-based assay, which persisted throughout the experiment, i.e., over 4 months. Titers did not differ between DTA mice and controls (Fig. 2B-D).

Comparison of multidimensional behavioral readouts among DTA and control groups with or without NMDAR1-AB

Testing mice in a multifaceted battery should reveal behavioral domains affected by the induced gray-matter encephalitis and shaped by NMDAR1-AB (Fig. 2E-R; Table 1). Indeed, spontaneous home cage behavior, measured by LABORAS, and corner visits as activity readout in the IntelliCage setup, revealed considerable hyperactivity of the DTA mice, interpretable as psychosis-like behavior or "loss of inhibition" [47]. The hyperactivity measures substantially inter correlated and were more pronounced upon the presence of NMDAR1-AB (Fig. 2E-G). Additional evidence of pathological hyperactivity is presented in Table 1. In fact, this psychosis-like feature seemed to persevere, since also the final test performed before perfusion, complex wheel running, similarly indicated hyperactivity (Fig. $2 \mathrm{H}$ ).

For hippocampal learning and memory, the classical Morris water maze (MWM) test was employed [48]. Whereas the visible platform days showed comparable ability of task learning among groups (Fig. 2l-K), substantial deficits arose regarding the hidden platform learning curves (Fig. 2L) and the probe trial results (Fig. 2M). Here, DTA mice, independent of the presence of NMDAR1-AB, demonstrated inferior performance consistent with hippocampal damage. This was also seen as a clear tendency in MWM reversal testing as hippocampus-dependent cognitive flexibility measure, again without an appreciable NMDAR1-AB effect (Fig. 2N-O).

Prepulse inhibition of the startle response (PPI) was employed as a surrogate marker for gating defects in the prefrontal cortex-known also as a highly relevant translational test in human patients with psychosis. PPI deficit is among the most reliable objective features of severe neuropsychiatric phenotypes, likely affecting the ability to adequately filter and interpret environmental stimuli $[47,49-52]$. Indeed, DTA mice, again independent of NMDAR1-AB, showed an overall tendency of a PPI deficit, pointing to prefrontal cortical network dysfunction as a consequence of the induced pyramidal neuronal death (Fig. 2P).

To some surprise, our previously designed extensive cognitive, emotional, and social phenotyping of mice in an observerindependent setting, using IntelliCages [29], did not reveal any considerable differences, except for the above-delineated distinct hyperactivity. This is most likely explained by lower sensitivity or ceiling effects of this paradigm (requiring a higher level of damage to show impairment), and the just partial involvement of hippocampal functions that are apparently fully compensated (example of IntelliCage readouts in Fig. 2Q, R). Similarly, all other behavioral tests failed to show appreciable alterations. Few just marginally significant and sometimes rather counter intuitive results proved invalid upon multiple-testing correction (Table 1 ). In conclusion, behavioral testing uncovered the expected consequences of pyramidal neuronal degeneration, i.e., substantial hippocampal dysfunction (MWM), a strong tendency of gating deficits as prefrontal cortex measure (PPI), and lasting psychosislike hyperactivity, with only the latter augmented by the presence of NMDAR1-AB. 


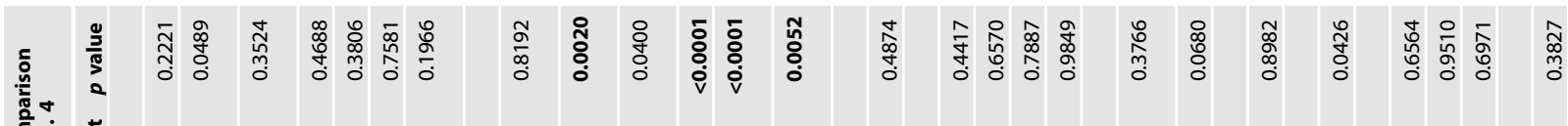
हु

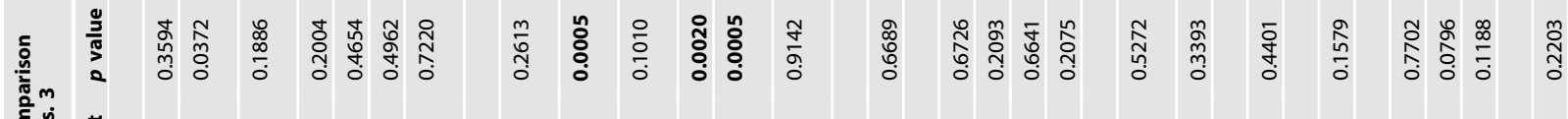
हैं

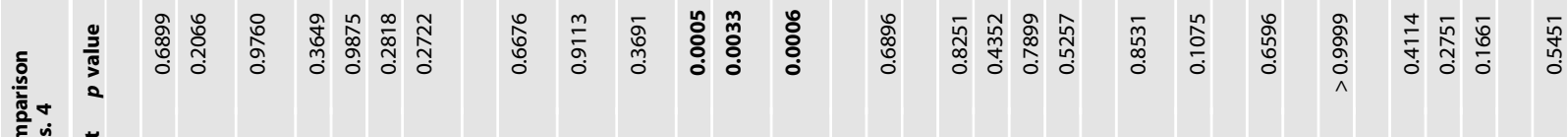
)

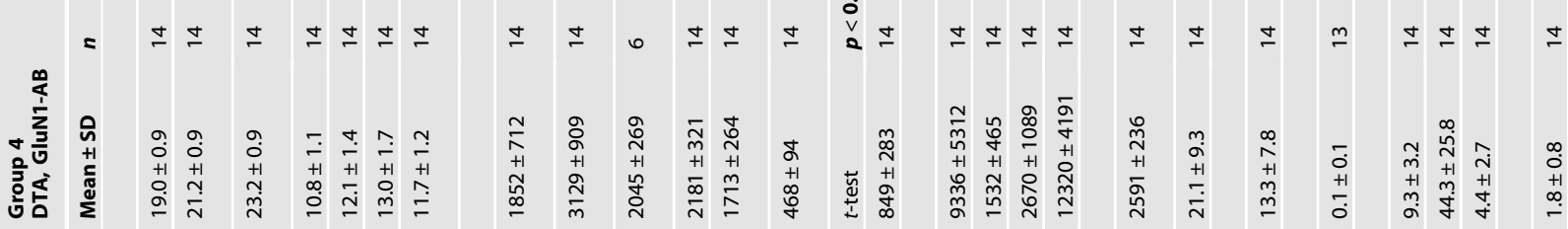

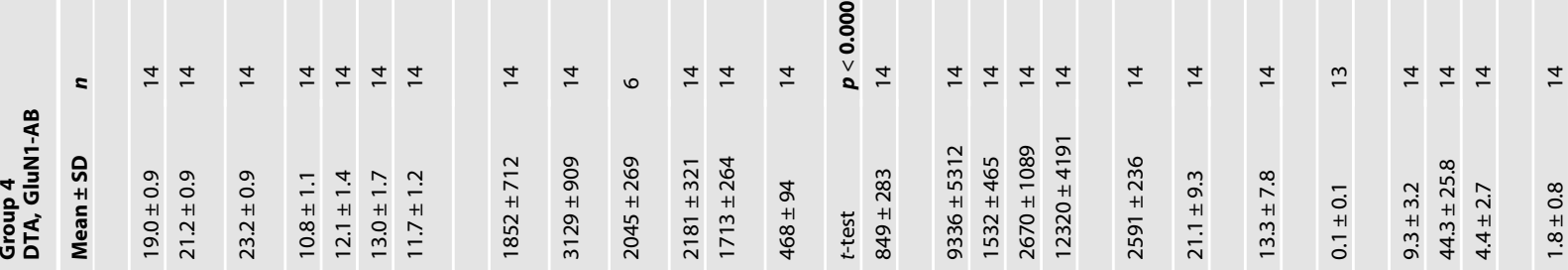

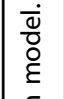

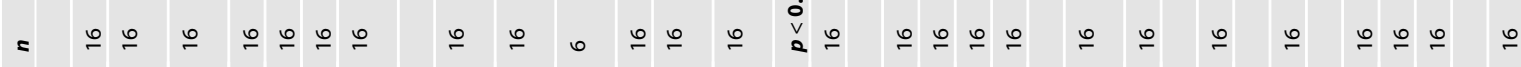

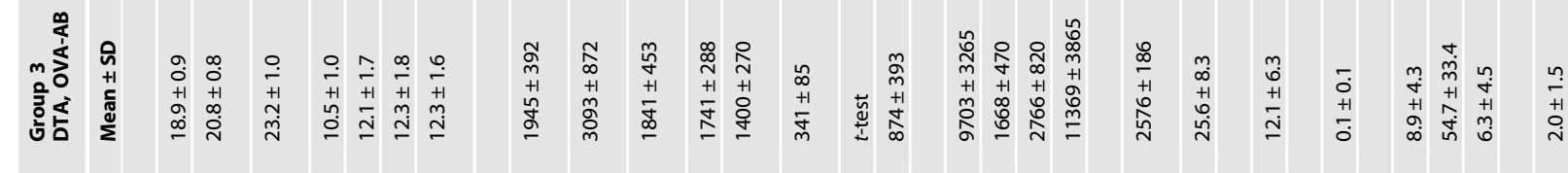

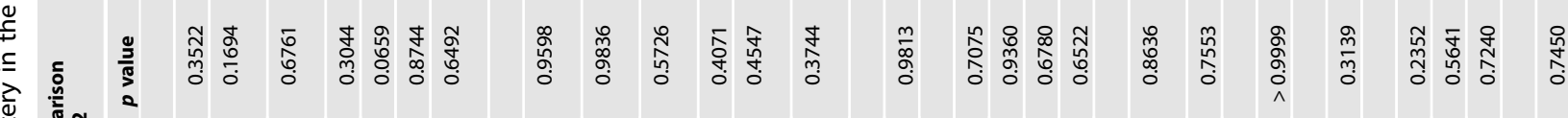
हैं

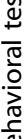

$m$
$\frac{1}{j}$
$\frac{1}{5}$

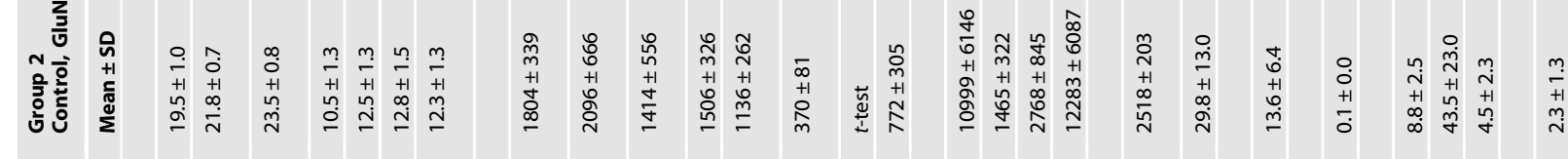
$\frac{\overbrace{}}{\frac{\pi}{\pi}}$

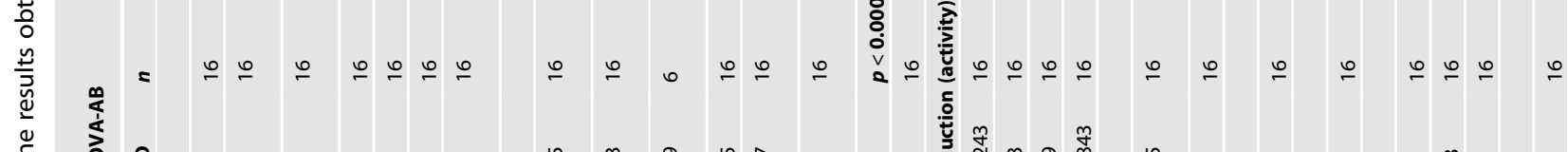

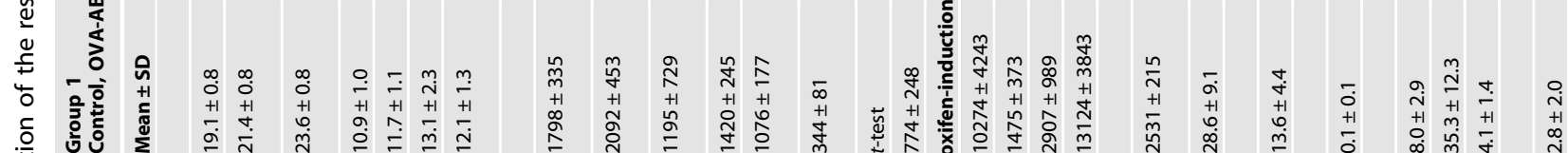
亮

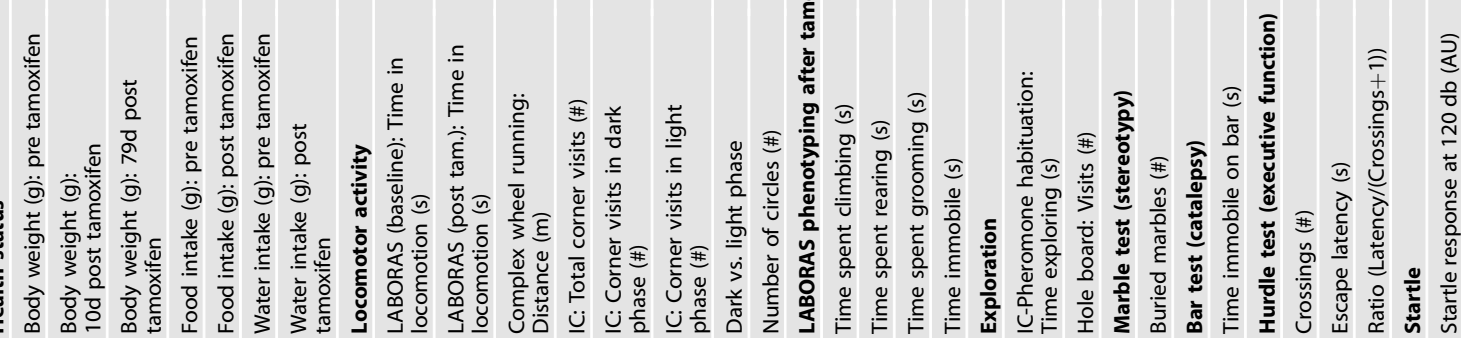




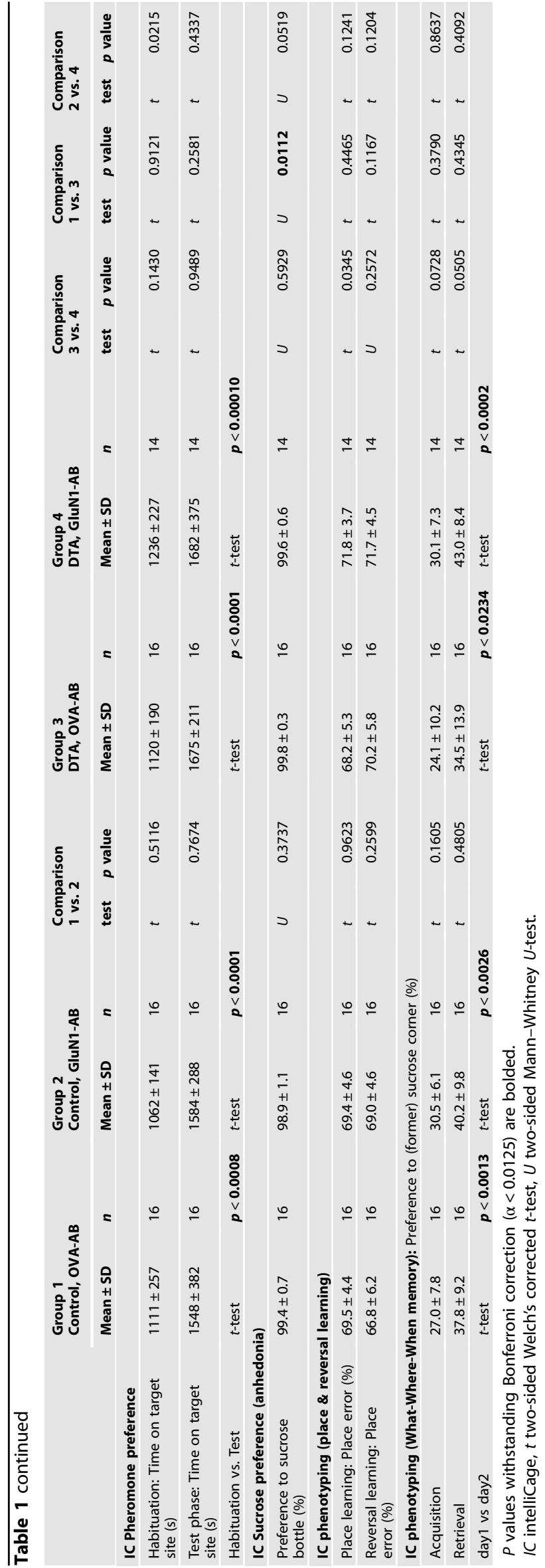

BBB integrity and measures of chronic inflammation in DTA and control groups with or without NMDAR1-AB

Using fluorescent tracers, as described in detail previously [28, 42], we addressed BBB leakiness in our DTA encephalitis model as a prerequisite for substantial NMDAR1-AB transfer to the brain and exertion of measurable effects. Tracer extravasation was enhanced for Evans blue and displayed a strong trend for fluorescein, confirming persistent BBB dysfunction. Brain water-content data showed remarkable scatters but were comparable in DTA and control mice at 3 months after encephalitis induction (Fig. 3A-D).

The chronic microglia and astrocyte response after tamoxifeninduced neuronal death in DTA mice was slightly milder as compared with the acute situation (see Fig. 1C, D), but still very obvious already from overview images (Fig. 3E, F). Histological quantification yielded distinct reductions of the areas of whole hippocampus, CA1 and CA3, whereas the dentate gyrus just revealed a similar tendency (Fig. 4A). Both Iba1 and GFAP quantifications followed the same pattern: strong microgliosis and astrogliosis in the whole hippocampus, CA1 and CA3, but only moderate in dentate gyrus of both DTA groups, with no significant differences in NMDAR1-AB carriers (Fig. 4B, C). NMDAR1immunized control mice showed no signs of inflammation as reported previously [28]. Change of inhibition could be demonstrated by the relative increases in parvalbumin-positive interneurons upon DTA-induced encephalitis, sparing the dentate gyrus. Also regarding these interneurons, NMDAR1-AB presence did not modulate the ensuing picture (Fig. 4D, E). Hippocampal immune cell infiltration, determined by counting CD45 + cells, was modest but clear in DTA mice (Fig. 4F). Collectively, these data document persistent BBB disruption and distinct chronic inflammation in our DTA model but no obvious amplifying influence of NMDAR1-AB on any of these readouts. This additionally argues against any appreciable proinflammatory role of NMDAR1-AB of the IgG class.

\section{Failure to induce any signal of encephalitis by immunizing} mice solely against the NMDAR1-N368/G369 region

During performance of the present work, a report claimed that immunizing mice against the NMDAR1-N368/G369 region alone induced an encephalopathy with remarkable B-cell response, provoking an autoimmune reaction against NMDAR, and encephalitis-like behavioral deficiencies [17]. We were first excited and wondered whether this model would finally help us to answer several burning questions of our own research, addressing for instance, the cellular-molecular mechanisms of NMDAR1-ABmediated brain inflammation, which we had not been able to observe [28], or late consequences of an autoimmune encephalitis, or reasons for NMDAR1-AB titer fluctuations including the occurence and nature of late boosters [53]. Therefore, we started an extensive experiment, ultimately using two large independent cohorts of wild-type mice, and followed meticulously this report's protocol [17]. However, as detailed in Fig. 5 and Table 2, none of the described findings could be reproduced, although the adopted immunization protocol worked well. We found specific GluN1 $1_{359-378-A B}$ of the lgG class in serum and no immune response using the non immunogenic control peptide GluN1 ${ }_{168}$ 187 (Fig. 5A-D). There was no BBB disturbance (Fig. 5E), all behavioral tests were normal (Fig. 5F-I), histology did not show any abnormalities (Fig. 5J-L), and brain FACS results were physiological and did not reveal any differences between groups (Fig. 5M-R). Therefore, a second mouse cohort was employed to exclude potential by-chance failure of replication but, disappointingly, yielded the same overall negative results (data not shown).

Simultaneous with our anticlimactic replication failure, Ding et al. published a study, using a similar immunization strategy to investigate the pathogenicity of various GluN1 peptides (each also at a dose of $200 \mu \mathrm{g}$ ), including the GluN1 $1_{359-377}$. Yet, in contrast to the claims of Wagnon et al., the authors did not find GluN1 $1_{359-377^{-}}$ 
A Experimental outline B Fore- Cere- C Fore- Cere- D Fore- Cere-

Tamoxifen

(2x i.p. $100 \mathrm{mg} / \mathrm{kg}$ )

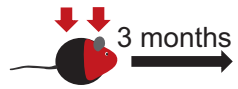

8 week-old control mice
BBB integrity test \& histology brain bellum brain bellum

E Persistent microglia response after tamoxifen-induced neuronal death in DTA mice

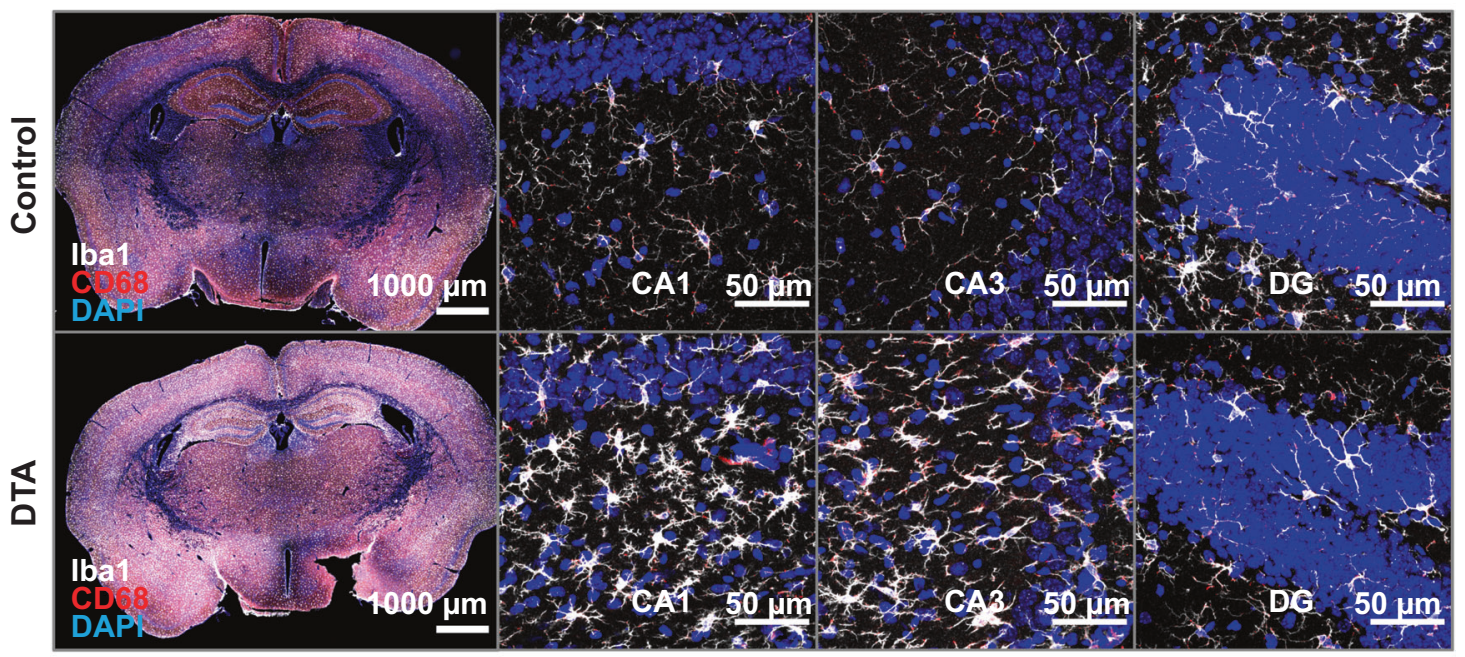

F Persistent astrocyte response after tamoxifen-induced neuronal death in DTA mice

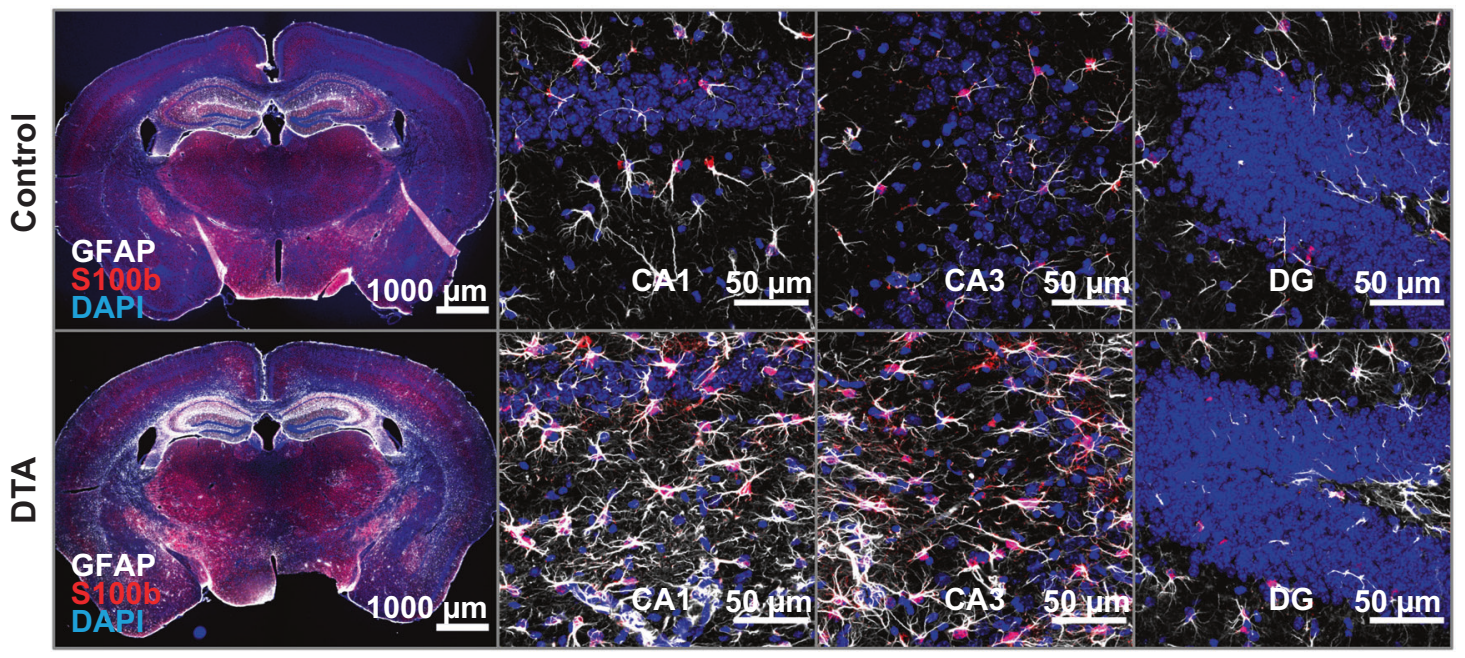

Fig. 3 Long-lasting inflammatory response at 3 months after tamoxifen induction. A Abbreviated experimental outline (detailed schematic in Fig. 2A). Blood-brain barrier (BBB) permeability, irrespective of NMDAR1-AB status, assessed by Evans blue (B) and fluorescein (C) extravasation, as well as brain-water content (D) in the forebrain and cerebellum of DTA and control mice. Data presented as mean \pm SD. E, F Representative images demonstrating persistent inflammatory changes in the hippocampus of DTA mice, including increased microglia density and apparent changes in morphology (E, quantifications in Fig. 4B) and increased expression of glial fibrillary acidic protein, GFAP (F), quantified in Fig. 4C). High-resolution images of CA1, CA3, and dentate gyrus (DG) regions were acquired as $10 \mu \mathrm{m}$ Z-stacks and displayed as maximum-intensity projections.

$A B$ in the CSF of immunized mice [54]. In addition, these authors validated the functionality of GluN1 $1_{356-385}$-specific $A B$ and assessed the in vivo consequences after triple immunization in combination with pertussis toxin, resulting in a behavioral phenotype (impaired social memory and novel object recognition, normal anxiety-, and depressive-like behavior), distinct from the one reported by Wagnon et al. (impaired spatial memory, abnormal anxiety-, and depressive-like behavior) [17, 54]. Unfortunately, Ding et al. did not investigate histopathological consequences of their immunization strategy, hence, it remains unclear whether they induced any features of an encephalitis. Another active immunization model, lately published as preprint, focused 


\section{Whole \\ hippocampus \\ CA1 \\ CA3 \\ Dentate gyrus}

A

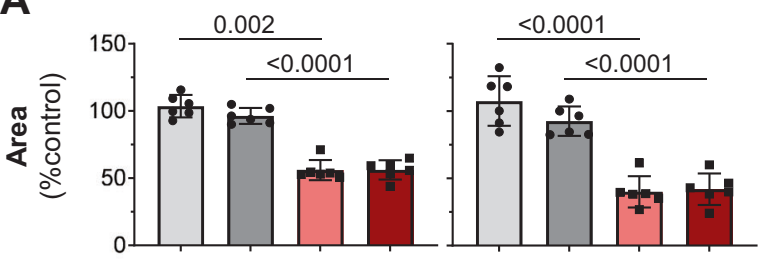

B

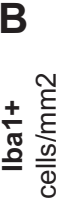
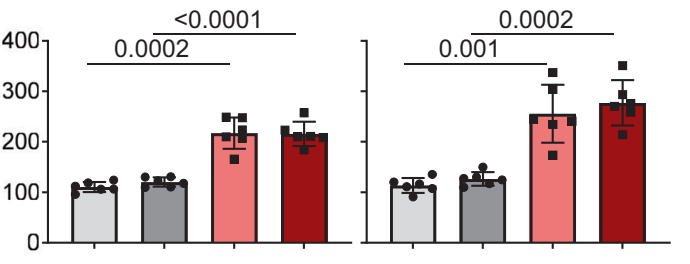

$c$

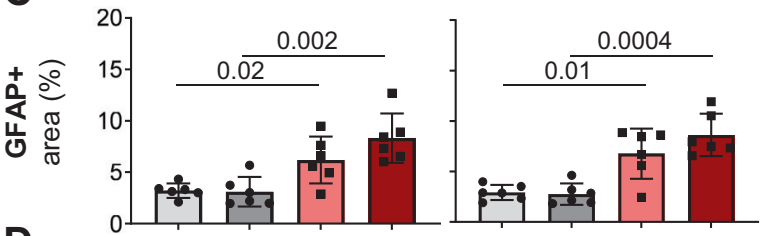

D
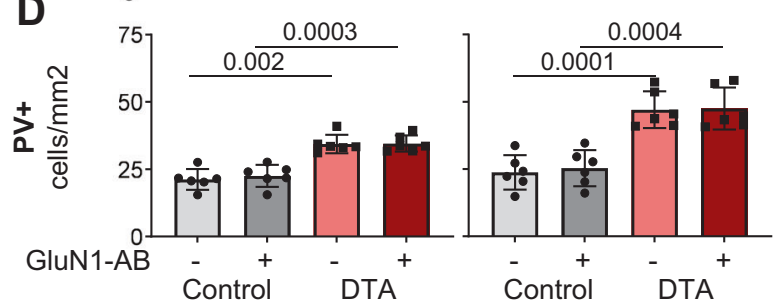

E

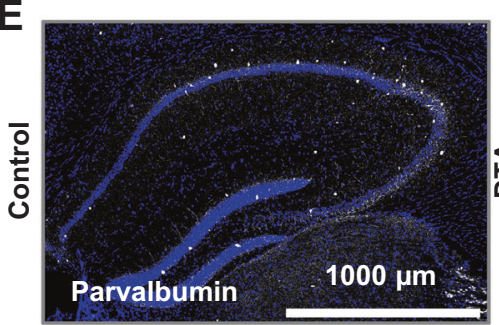

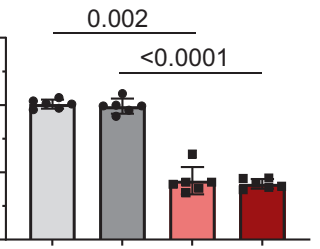
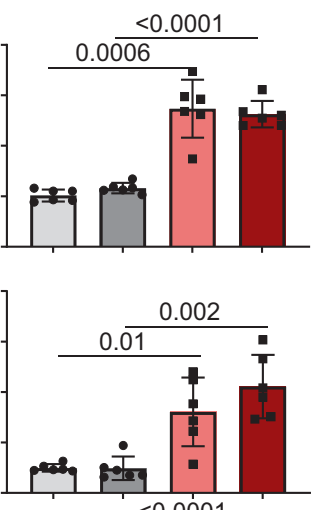

$<0.0001$

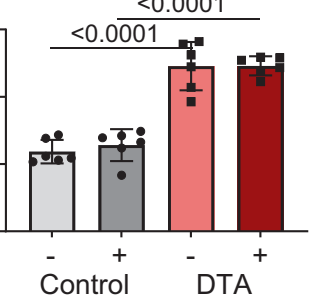

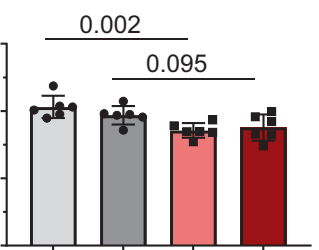
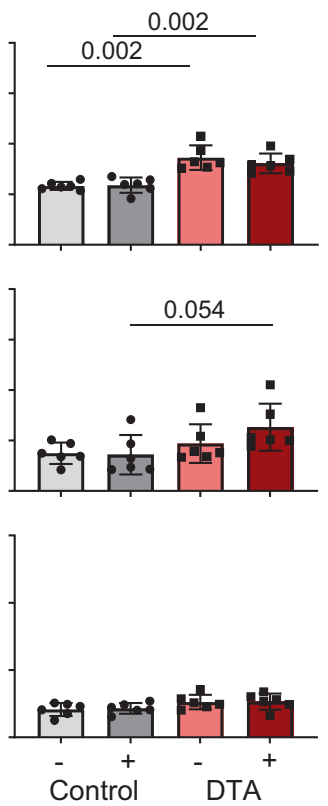

F Whole hippocampus
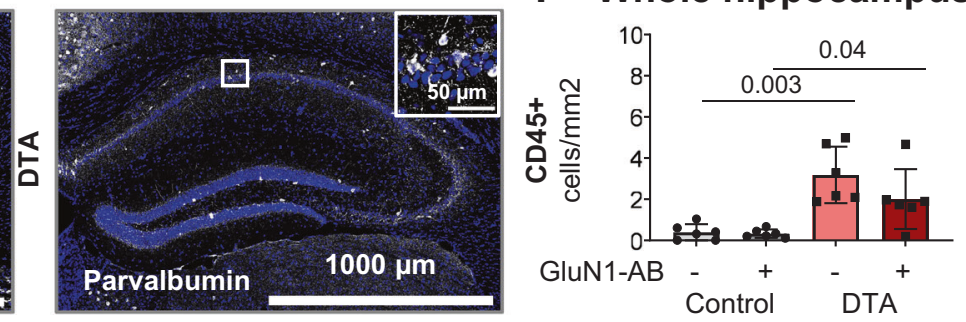

Fig. 4 Histological quantification of typical encephalitis readouts in the hippocampus. A For assessment of atrophy, the respective hippocampal areas were normalized to the mean of control mice. Normalized area shows the average of 6 sections per mouse (within Bregma $-1.34 \mathrm{~mm}$ and $-1.64 \mathrm{~mm}$ ). B Number of Iba1+ cells (microglia). C GFAP+ area determined densitometrically upon uniform thresholding. D Density of parvalbumin (PV)-positive interneurons. E Representative images of parvalbumin stainings in control and DTA mice. F Leukocyte $\left(\mathrm{CD} 45+\right.$ and $\mathrm{lba} 1-$ cells $\left./ \mathrm{mm}^{2}\right)$ infiltration into the hippocampal parenchyma. Data from 6 mice/group displayed as mean \pm SD.

on the chronic effects of NMDAR1-AB upon immunization against GluN1 $1_{402-421}$ peptide. Similar to our experience with these immunizations, mice remained healthy and did not develop any encephalitic signs, despite the presence of persistent and functional NMDAR1-AB. The only behavioral alteration reliably observed in GluN1 ${ }_{402-421}$-immunized mice was impaired spatial memory and/or novelty recognition, assessed as spontaneous alternation in T-maze [55]. These findings are highly interesting since they underscore the possibility that chronic presence of circulating NMDAR1-AB can mildly modulate behavior, even in the absence of an appreciable BBB dysfunction. This is in line with a previous expert review [18] and our experimental observation that NMDAR1-AB can reach the brain to bind there at low titers even in healthy wild-type mice [26].

We are aware that-for comparison-in experimental autoimmune encephalitis (EAE), fluctuations can occur regarding the severity of the clinical/pathological picture [56-58]; however, the absence of all claimed readouts as obtained in our replication approaches, is unheard of and quite surprising. This is more so, since the message of the report by Wagnon et al. will remain, if not questioned, and be taken for granted. This in turn can become an ethical issue, leading to (pre)clinical conclusions that are ultimately damaging for other scientists and unfortunately also for patients, as we observe on a regular basis.

\section{Conclusions from the NMDAR1-immunized DTA model: NMDAR1-AB modify rather than cause an encephalitis} Using a well-standardizable, spatially and temporally defined mouse model of viral encephalitis by employing controlled DTA induction in pyramidal neurons, we find a multifaceted encephalitic phenotype, which persists over months. This phenotype involves pyramidal neurons and thus of course also their NMDAR, but is only marginally aggravated in NMDAR1-AB carriers versus non carriers. The aggravation essentially rests on hyperactivity as 
A Experimental outline (according to Wagnon et al 2020)

Experimental groups:

WT-OVA

WT-GluN1 $1_{359-378}$

\begin{tabular}{|c|c|}
\hline $\begin{array}{l}\text { Immunization } \\
\text { age: } 8 \text { weeks } \\
\text { day } 0\end{array}$ & $\begin{array}{c}\text { Pertussis } \\
\text { toxin } \\
\text { day } 2\end{array}$ \\
\hline
\end{tabular}

\begin{tabular}{|c|c} 
Open field \\
day 13-14 maze \\
day 14+15
\end{tabular}

\begin{tabular}{|c|c|}
$\begin{array}{c}\text { Elevated } \\
\text { plus maze } \\
\text { day } 16\end{array}$ & $\begin{array}{c}\text { Forced } \\
\text { swim test } \\
\text { day } 17\end{array}$ \\
\hline
\end{tabular}

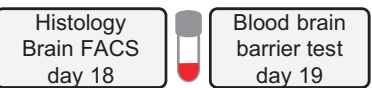

B

OVA ELISA

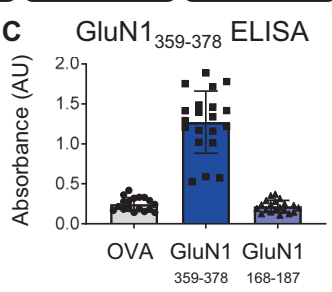

D GluN1 $168-187$ ELISA

E
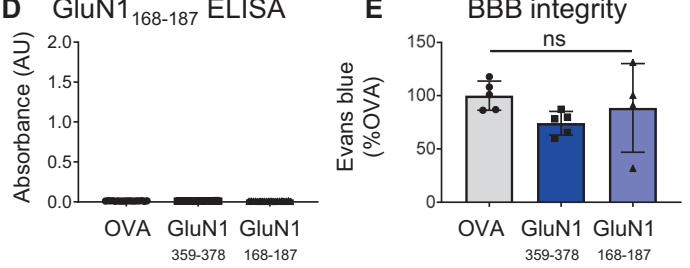

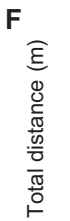

G Elevated plus maze

H Elevated plus maze

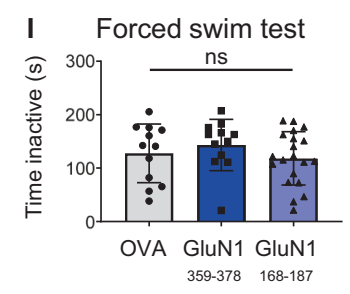

J Histology - Microglia

L
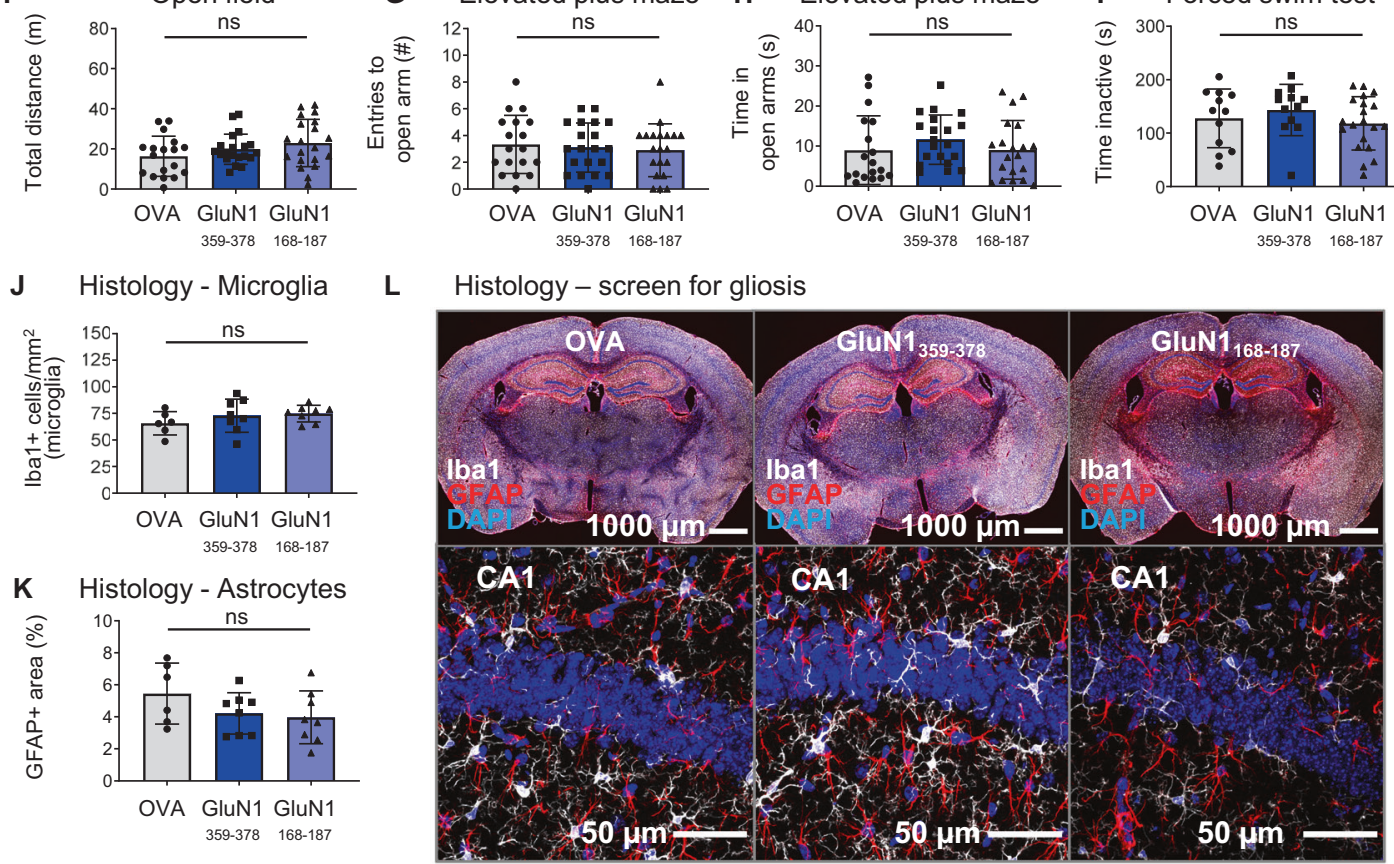

M Brain FACS Gating Strategy

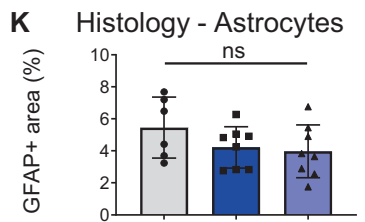

OVA GluN1 GluN1 359-378 $\quad 168-187$
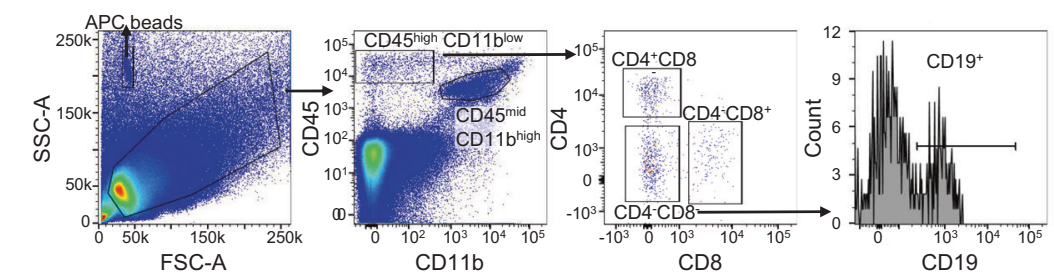

N Brain FACS - Microglia

O Brain FACS - Leucocytes

P T-cells in CD45 high CD11blow
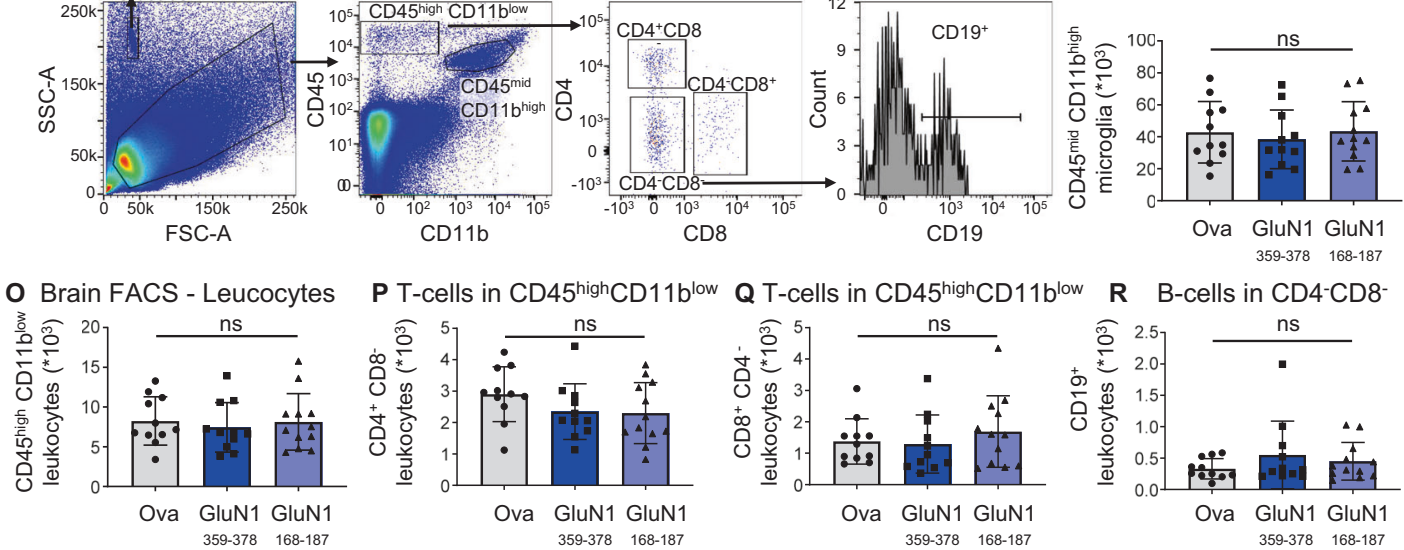

Fig. 5 Summary of the results from the replication study. A Experimental outline, following the protocol of Wagnon et al. [17]. B-D Experimental validation of immunization success using OVA-ELISA, GluN1 $1_{359-378}$-ELISA, and GluN1 ${ }_{168-187}$-ELISA. E Blood-brain-barrier (BBB) integrity assessed through Evans blue extravasation. F-I Results of behavioral phenotyping, showing locomotor activity in the open field, anxiety-related behavior in elevated plus maze, and depression-like behavior in the forced-swim test. J-L Histological quantification using 8 mice/immunization with focus on reactive gliosis, showing microglia numbers, GFAP+ area (densitometry), and representative images of quantified stainings. High-resolution images of CA1 were acquired as $10 \mu \mathrm{m}$ Z-stacks and displayed as maximum-intensity projections. M-R Characterization of the brains' immune cell compartment by flow cytometry of 11-12 mice/group. M Gating strategy. Quantification of $\mathrm{CD} 11 \mathrm{~b}^{\text {high }} \mathrm{CD} 45^{\text {mid }}$ cells (microglia). Quantification of $\mathrm{CD} 11 \mathrm{~b}^{\text {low }} \mathrm{CD}^{\mathrm{h}} 5^{\text {high }}$ leukocytes, CD4 ${ }^{+} \mathrm{T}$ cells, CD8 ${ }^{+} \mathrm{T}$ cells, and CD19 ${ }^{+} \mathrm{B}$ cells. Data displayed as mean \pm SD. 
Table 2. Detailed presentation of behavior results obtained in replication study (protocol following Wagnon et al. [17]).

\begin{tabular}{|c|c|c|c|c|c|c|c|c|}
\hline & \multicolumn{2}{|l|}{ OVA } & \multicolumn{2}{|l|}{ GluN1 } & \multicolumn{2}{|l|}{ GluN1 168-187 } & \multicolumn{2}{|c|}{ ANOVA } \\
\hline & Mean \pm SD & $n$ & Mean \pm SD & $n$ & Mean \pm SD & $n$ & test & $p$ value \\
\hline \multicolumn{9}{|l|}{ Health status } \\
\hline Pre immune: Body weight (g) & $24.2 \pm 1.0$ & 18 & $24.5 \pm 0.9$ & 20 & $24.6 \pm 1.1$ & 20 & 1way & 0.4515 \\
\hline Post immune $(+7 d)$ : Body weight $(g)$ & $23.3 \pm 1.2$ & 18 & $23.0 \pm 0.8$ & 20 & $23.4 \pm 1.0$ & 20 & 1 way & 0.3503 \\
\hline \multicolumn{9}{|l|}{ Depression-like behavior } \\
\hline FST total time immobile (s) & $127.8 \pm 54.9$ & 12 & $143.4 \pm 48.0$ & 12 & $118.2 \pm 49.8$ & 20 & 1way & 0.4048 \\
\hline Open field: Total distance $(\mathrm{m})$ & $16.3 \pm 10.0$ & 18 & $19.8 \pm 7.5$ & 20 & $23.0 \pm 11.8$ & 20 & 1way & 0.1284 \\
\hline \multicolumn{9}{|l|}{ Activity } \\
\hline Elevated plus maze: Total arm visits (\#) & $11.4 \pm 4.0$ & 18 & $11.7 \pm 4.7$ & 20 & $10.5 \pm 5.1$ & 20 & KW & 0.8547 \\
\hline Y maze (habituation): Total arm visits (\#) & $10.0 \pm 4.0$ & 18 & $13.3 \pm 5.8$ & 19 & $13.9 \pm 6.9$ & 19 & 1 way & 0.0956 \\
\hline \multicolumn{9}{|l|}{ Anxiety-related behavior } \\
\hline Elevated plus maze: Visits to open arm (\#) & $3.3 \pm 2.2$ & 18 & $3.1 \pm 1.8$ & 20 & $2.9 \pm 2.0$ & 20 & 1 way & 0.7994 \\
\hline Elevated plus maze: Time in open arm (s) & $9.0 \pm 8.5$ & 18 & $11.6 \pm 6.1$ & 20 & $9.1 \pm 7.3$ & 20 & KW & 0.2046 \\
\hline \multicolumn{9}{|l|}{ Y maze (habituation, $5 \mathrm{~min}$ ) } \\
\hline Y maze: Visits arm 1 (\#) & $4.4 \pm 2.4$ & 18 & $6.4 \pm 3.5$ & 19 & $7.4 \pm 4.5$ & 19 & KW & 0.1004 \\
\hline Y maze: Visits arm 2 (\#) & $5.6 \pm 2.5$ & 18 & $6.9 \pm 3.0$ & 19 & $6.5 \pm 2.9$ & 19 & 1 way & 0.3567 \\
\hline arm1 vs. arm2 & $U$ test & $p=0.1120$ & $t$ test & $p=0.5860$ & $t$ test & $p=0.4426$ & & \\
\hline \multicolumn{9}{|l|}{$Y$ maze (test phase, $3 \mathrm{~min}$ ) } \\
\hline Y maze: Visits arm 1 (\#) & $1.9 \pm 1.6$ & 18 & $2.5 \pm 1.8$ & 19 & $2.1 \pm 2.1$ & 19 & KW & 0.5813 \\
\hline Y maze: Visits arm 2 (\#) & $2.4 \pm 2.6$ & 18 & $2.6 \pm 1.7$ & 19 & $2.1 \pm 1.2$ & 19 & KW & 0.4752 \\
\hline Y maze: Visits novel arm (\#) & $2.8 \pm 1.7$ & 18 & $3.5 \pm 2.2$ & 19 & $3.3 \pm 1.8$ & 19 & KW & 0.5482 \\
\hline
\end{tabular}

Significant $p$ values $(\alpha<0.05)$ in bold.

FST forced swim test, 1 way one-way ANOVA; KW Kruskal-Wallis test, $t$ two-sided Welch's corrected $t$-test, $U$ two-sided Mann-Whitney $U$-test.

psychosis-like behavioral readout but is not reflected in any histological quantification. Similarly, comparison of NMDAR1-ABpositive and -negative human encephalitis cases did not reveal appreciable differences, except for few NMDAR-antagonistic (ketamine-like) symptoms [14]. Most likely, human "NMDAR encephalitis" is simply not a separate condition, but rather marks an encephalitis where the highly prevalent NMDAR1-AB and/or the respective $B$ cells happen to be present in the brain and shape the clinical picture. Therefore, it may be problematic, if the search for encephalitis causes stops after detection of NMDAR1-AB (of the IgG class). Reassuringly, "polypragmatic" treatment of any encephalitis of unknown origin (constituting the majority of cases) should anyhow include antibiotics, antivirals, and eventually corticosteroids/immunosuppressants on top of supporting measures.

The N-terminal domain containing the G7 epitope (N368/G369) was first deemed pathognomonic for "NMDAR encephalitis," and believed to be the target region of the pathological NMDAR1-AB of the lgG class seen responsible for this condition [59]. Therefore, the respective immunization model, leading to encephalitis as described by Wagnon and colleagues [17], seemed attractive at first view and worthwhile pursuing. Unfortunately, it was not reproducible in our hands, and is not supported by a similar recent paper [54]. Searching for an explanation by speculating about possible reasons why Wagnon and colleagues found signs of an encephalitis that we did not see, interfering "iatrogenic" issues may be worthwhile considering, like e.g., undetected subclinical infections in their animal facility, leading to "occult" brain inflammation. Also, other factors, e.g., differences in the gut microbiota, which have the potential to modulate disease progression in EAE models [60], cannot be entirely excluded. However, it seems rather unlikely that the overall discrepancy, including BBB dysfunction, can be explained by such physiological factors.

Considering our own previous findings on epitopes recognized by the highly frequent NMDAR1-AB found in human serum, the negative outcome of the replication attempts is actually not too surprising. Epitope mapping using 7 different NMDAR1 constructs revealed recognition by NMDAR1-AB-positive sera of different epitopes, located in the extracellular ligand-binding and the $\mathrm{N}$ terminal domain, as well as the intracellular C-terminal and the extended pore domain. NMDAR1-AB seropositivity was polyclonal/ polyspecific in half of the investigated sera and likely mono- or oligoclonal/oligospecific (mainly lgG) in the other half. Overall, no particular disease-related pattern appeared. NMDAR1 epitopes were comparable across health and disease [61]. Also, the accentuated role of IgG in "NMDAR encephalitis" is still a matter of speculation, but likely related to inflammation-induced class 
switch in the brain [62]. As mentioned in the Introduction, NMDAR1-AB are only one of many possible autoantibodies directed against brain epitopes [18-22]. The finally resulting phenotype would then depend on (i) the specific site(s) of brain inflammation, with either resident plasma cells producing $A B$ or a local extent of $B B B$ breach at that site (to allow sufficient $A B$ transfer to the brain), and (ii) the specific circulating brain-reactive autoantibody profile of each individual.

To conclude, while NMDAR1-AB can contribute to the behavioral phenotype of an underlying encephalitis, there is no proof at present for induction of an encephalitis by NMDAR1-AB themselves. Thus, based on the results presented here, the answer to the question of whether or not NMDAR1-AB can, by themselves, induce encephalitis is probably no, with the caveat that perhaps it may be possible in extremely rare patients with an exceptionally high NMDAR1-AB titer. However, this would assume that a very high titer is somehow linked to brain inflammation (as cause or consequence) and/or local increases in BBB permeability, which is neither supported by the data from this nor from other studies.

\section{DATA AVAILABILITY}

All data are available upon request.

\section{REFERENCES}

1. Dalmau J, Gleichman AJ, Hughes EG, Rossi JE, Peng X, Lai M, et al. Anti-NMDAreceptor encephalitis: case series and analysis of the effects of antibodies. Lancet Neurol. 2008;7:1091-8.

2. Dalmau J, Tüzün E, Wu HY, Masjuan J, Rossi JE, Voloschin A, et al. Paraneoplastic anti-N-methyl-D-aspartate receptor encephalitis associated with ovarian teratoma. Ann Neurol. 2007;61:25-36.

3. Hughes EG, Peng X, Gleichman AJ, Lai M, Zhou L, Tsou R, et al. Cellular and synaptic mechanisms of anti-NMDA receptor encephalitis. J Neurosci. 2010;30:5866-75.

4. Manto M, Dalmau J, Didelot A, Rogemond V, Honnorat J. In vivo effects of antibodies from patients with anti-NMDA receptor encephalitis: further evidence of synaptic glutamatergic dysfunction. Orphanet J Rare Dis. 2010;5:1-12.

5. Mikasova L, De Rossi P, Bouchet D, Georges F, Rogemond V, Didelot A, et al. Disrupted surface cross-talk between NMDA and Ephrin-B2 receptors in antiNMDA encephalitis. Brain 2012;135:1606-21.

6. Hammer C, Stepniak B, Schneider A, Papiol S, Tantra M, Begemann M, et al. Neuropsychiatric disease relevance of circulating anti-NMDA receptor autoantibodies depends on blood-brain barrier integrity. Mol Psychiatry. 2014;19:1143-9.

7. Wright S, Hashemi K, Stasiak L, Bartram J, Lang B, Vincent A, et al. Epileptogenic effects of NMDAR antibodies in a passive transfer mouse model. Brain 2015;138:3159-67.

8. Li Y, Tanaka K, Wang L, Ishigaki $Y$, Kato N. Induction of memory deficit in mice with chronic exposure to cerebrospinal fluid from patients with Anti-N-Methyl-DAspartate receptor encephalitis. Tohoku J Exp Med. 2015;237:329-38.

9. Würdemann T, Kersten M, Tokay T, Guli X, Kober M, Rohde M, et al. Stereotactic injection of cerebrospinal fluid from anti-NMDA receptor encephalitis into rat dentate gyrus impairs NMDA receptor function. Brain Res. 2016;1633:10-8.

10. Planagumà J, Leypoldt F, Mannara F, Gutiérrez-Cuesta J, Martín-García E, Aguilar $\mathrm{E}$, et al. Human N-methyl D-aspartate receptor antibodies alter memory and behaviour in mice. Brain 2015;138:94-109.

11. Blome R, Bach W, Guli X, Porath K, Sellmann T, Bien CG, et al. Differentially altered NMDAR dependent and independent long-term potentiation in the CA3 subfield in a model of anti-NMDAR encephalitis. Front Synaptic Neurosci. 2018;10:1-13.

12. Kersten $M$, Rabbe $T$, Blome $R$, Porath $K$, Sellmann $T$, Bien CG, et al. Novel object recognition in rats with NMDAR dysfunction in CA1 after stereotactic injection of anti-NMDAR encephalitis cerebrospinal fluid. Front Neurol. 2019;10:1-11.

13. Taraschenko O, Fox HS, Pittock SJ, Zekeridou A, Gafurova M, Eldridge E, et al. A mouse model of seizures in anti-N-methyl-d-aspartate receptor encephalitis. Epilepsia. 2019;60:452-63.

14. Chen X, Li JM, Liu F, Wang Q, Zhou D, Lai X. Anti-N-methyl-D-aspartate receptor encephalitis: a common cause of encephalitis in the intensive care unit. Neurol Sci. 2016;37:1993-8.

15. Jones BE, Tovar KR, Goehring A, Jalali-Yazdi F, Okada NJ, Gouaux E, et al. Autoimmune receptor encephalitis in mice induced by active immunization with conformationally stabilized holoreceptors. Sci Transl Med. 2019;11: eaaw0044.

16. Ehrenreich $\mathrm{H}$, Pan $\mathrm{H}$, Hollmann M. RE: Active immunization, autoimmunity and encephalitis: the missing links. Sci Transl Med. 2019. https://stm.sciencemag.org/ content/11/500/eaaw0044/tab-e-letters.

17. Wagnon I, Hélie $P$, Bardou I, Regnauld C, Lesec L, Leprince J, et al. Autoimmune encephalitis mediated by $\mathrm{B}$-cell response against $\mathrm{N}$-methyl-d-aspartate receptor. Brain. 2020;143:2957-72

18. Diamond B, Huerta PT, Mina-Osorio P, Kowal C, Volpe BT. Losing your nerves? Maybe it's the antibodies. Nat Rev Immunol. 2009;9:449-56.

19. Crisp SJ, Kullmann DM, Vincent A. Autoimmune synaptopathies. Nat Rev Neurosci. 2016;17:103-17.

20. Nagele EP, Han M, Acharya NK, DeMarshall C, Kosciuk MC, Nagele RG. Natural IgG autoantibodies are abundant and ubiquitous in human sera, and their number is influenced by age, gender, and disease. PLoS One. 2013;8:e60726.

21. Pollak TA, Lennox $B R$, Müller $S$, Benros $M E$, Prüss $H$, Tebartz van Elst $L$, et al. Autoimmune psychosis: an international consensus on an approach to the diagnosis and management of psychosis of suspected autoimmune origin. Lancet Psychiatry. 2020;7:93-108.

22. Cohen IR, Young DB. Autoimmunity, microbial immunity and the immunological homunculus. Immunol Today. 1991;12:105-10.

23. Prüss H, Finke $C$, Höltje $M$, Hofmann J, Klingbeil C, Probst $C$, et al. N-methyl-Daspartate receptor antibodies in herpes simplex encephalitis. Ann Neurol. 2012;72:902-11.

24. Armangue T, Moris G, Cantarín-Extremera V, Conde CE, Rostasy K, Erro ME, et al. Autoimmune post-herpes simplex encephalitis of adults and teenagers. Neurology. 2015;85:1736-43.

25. Pan H, Steixner-Kumar AA, Seelbach A, Deutsch N, Ronnenberg A, Tapken D, et al. Multiple inducers and novel roles of autoantibodies against the obligatory NMDAR subunit NR1: a translational study from chronic life stress to brain injury. Mol Psychiatry. 2020. https://doi.org/10.1038/s41380-020-0672-1.

26. Castillo-Gomez E, Kästner A, Steiner J, Schneider A, Hettling B, Poggi G, et al. The brain as immunoprecipitator of serum autoantibodies against N-Methyl-Daspartate receptor subunit NR1. Ann Neurol. 2016;79:144-51.

27. Dalmau J, Armangué T, Planagumà J, Radosevic M, Mannara F, Leypoldt $F$, et al. An update on anti-NMDA receptor encephalitis for neurologists and psychiatrists: mechanisms and models. Lancet Neurol. 2019;18:1045-57.

28. Pan H, Oliveira B, Saher G, Dere E, Tapken D, Mitjans M, et al. Uncoupling the widespread occurrence of anti-NMDAR1 autoantibodies from neuropsychiatric disease in a novel autoimmune model. Mol Psychiatry. 2019;24:1489-501.

29. Dere E, Ronnenberg A, Tampe B, Arinrad S, Schmidt M, Zeisberg E, et al. Cognitive, emotional and social phenotyping of mice in an observer-independent setting. Neurobiol Learn Mem. 2018;150:136-50.

30. Agarwal A, Dibaj P, Kassmann CM, Goebbels S, Nave KA, Schwab MH. In vivo imaging and noninvasive ablation of pyramidal neurons in adult NEX-CreERT2 mice. Cereb Cortex. 2012;22:1473-86.

31. Ivanova A, Signore M, Caro N, Greene ND, Copp AJ, Martinez-Barbera JP. In vivo genetic ablation by Cre-mediated expression of diphtheria toxin fragment $A$. Genes. 2005:43:129-35.

32. El-Kordi A, Winkler D, Hammerschmidt K, Kästner A, Krueger D, Ronnenberg A, et al. Development of an autism severity score for mice using Nlgn4 null mutants as a construct-valid model of heritable monogenic autism. Behav Brain Res. 2013;251:41-9.

33. Dere E, Dahm L, Lu D, Hammerschmidt K, Ju A, Tantra M, et al. Heterozygous ambra1 deficiency in mice: a genetic trait with autism-like behavior restricted to the female gender. Front Behav Neurosci. 2014;8:1-19.

34. Dere E, Winkler D, Ritter C, Ronnenberg A, Poggi G, Patzig J, et al. Gpm6b deficiency impairs sensorimotor gating and modulates the behavioral response to a 5-HT2A/C receptor agonist. Behav Brain Res. 2015;277:254-63.

35. Janova $H$, Arinrad S, Balmuth E, Mitjans M, Hertel J, Habes $M$, et al. Microglia ablation alleviates myelin-associated catatonic signs in mice. J Clin Invest. 2018;128:734-45.

36. Garcia-Agudo LF, Janova H, Sendler LE, Arinrad S, Steixner AA, Hassouna I, et al. Genetically induced brain inflammation by $\mathrm{Cnp}$ deletion transiently benefits from microglia depletion. FASEB J. 2019;33:8634-47.

37. Winkler D, Daher F, Wüstefeld L, Hammerschmidt K, Poggi G, Seelbach A, et al. Hypersocial behavior and biological redundancy in mice with reduced expression of PSD95 or PSD93. Behav Brain Res. 2018;352:35-45.

38. Netrakanti PR, Cooper BH, Dere E, Poggi G, Winkler D, Brose N, et al. Fast cerebellar reflex circuitry requires synaptic vesicle priming by munc13-3. Cerebellum. 2015;14:264-83.

39. Wakhloo D, Scharkowski F, Curto Y, Javed Butt U, Bansal V, Steixner-Kumar AA, et al. Functional hypoxia drives neuroplasticity and neurogenesis via brain erythropoietin. Nat Commun. 2020;11:1-12. 
40. Liebetanz D, Baier PC, Paulus W, Meuer K, Bahr M, Weishaupt JH. A highly sensitive automated complex running wheel test to detect latent motor deficits in the mouse MPTP model of Parkinson's disease. Exp Neurol. 2007;205:207-13.

41. McKenzie IA, Ohayon D, Li H, de Faria JP, Emery B, Tohyama K, et al. Motor skill learning requires active central myelination. Science. 2014;346:318-22.

42. Berghoff SA, Duking T, Spieth L, Winchenbach J, Stumpf SK, Gerndt N, et al. Blood-brain barrier hyperpermeability precedes demyelination in the cuprizone model. Acta Neuropathol Commun. 2017;5:1-13.

43. Schindelin J, Arganda-Carreras I, Frise E, Kaynig V, Longair M, Pietzsch T, et al. Fiji: an open-source platform for biological-image analysis. Nat Methods. 2012;9:676-82.

44. R Development Core Team. R: A language and environment for statistical computing. Vienna, Austria: R Foundation for Statistical Computing; 2020.

45. Kassambara A. rstatix: pipe-friendly framework for basic statistical tests. 0.7.0 ed 2021.

46. Brockschnieder D, Lappe-Siefke C, Goebbels S, Boesl MR, Nave KA, Riethmacher D. Cell depletion due to diphtheria toxin fragment $A$ after Cre-mediated recombination. Mol Cell Biol. 2004;24:7636-42.

47. Radyushkin K, El-Kordi A, Boretius S, Castaneda S, Ronnenberg A, Reim K, et al. Complexin2 null mutation requires a 'second hit' for induction of phenotypic changes relevant to schizophrenia. Genes Brain Behav. 2010;9:592-602.

48. Morris RGM. Spatial localization does not require the presence of local cues. Learn Motiv. 1981;12:239-60.

49. Braff D, Stone C, Callaway E, Geyer M, Glick I, Bali L. Prestimulus effects on human startle reflex in normals and schizophrenics. Psychophysiology. 1978;15:339-43.

50. Fendt $M$, Koch M. Translational value of startle modulations. Cell Tissue Res. 2013;354:287-95.

51. Kumari V, Das M, Zachariah E, Ettinger U, Sharma T. Reduced prepulse inhibition in unaffected siblings of schizophrenia patients. Psychophysiology. 2005;42: 588-94.

52. Poggi G, Boretius S, Möbius W, Moschny N, Baudewig J, Ruhwedel T, et al. Cortical network dysfunction caused by a subtle defect of myelination. Glia. 2016;64:2025-40.

53. Ehrenreich H, Wilke J, Steixner-Kumar AA. Spontaneous serum autoantibody fluctuations: To be or not to be. Mol Psychiatry. 2020. https://doi.org/10.1038/ s41380-020-00883-4.

54. Ding $Y$, Zhou Z, Chen J, Peng $Y$, Wang H, Qiu W, et al. Anti-NMDAR encephalitis induced in mice by active immunization with a peptide from the amino-terminal domain of the GluN1 subunit. Journal of Neuroinflammation. 2021;18:1-10.

55. Yue W, Caldwell S, Risbrough V, Powell S, Zhou X. Chronic Presence of Blood Circulating Anti-NMDAR1 Autoantibodies Impairs Cognitive Function in Mice. bioRxiv. 2021. https://doi.org/10.1101/2021.04.22.440976.

56. Glatigny S, Bettelli E. Experimental autoimmune encephalomyelitis (EAE) as animal models of multiple sclerosis (MS). Cold Spring Harb Perspect Med. 2018;8:1-19.

57. Gold $\mathrm{R}$, Linington $\mathrm{C}$, Lassmann $\mathrm{H}$. Understanding pathogenesis and therapy of multiple sclerosis via animal models: 70 years of merits and culprits in experimental autoimmune encephalomyelitis research. Brain 2006;129:1953-71.

58. Comabella M, Martin R. Genomics in multiple sclerosis-current state and future directions. J Neuroimmunol. 2007;187:1-8.

59. Gleichman AJ, Spruce LA, Dalmau J, Seeholzer SH, Lynch DR. Anti-NMDA receptor encephalitis antibody binding is dependent on amino acid identity of a small region within the GluN1 amino terminal domain. J Neurosci. 2012;32:11082-94.

60. Gandy KAO, Zhang J, Nagarkatti P, Nagarkatti M. The role of gut microbiota in shaping the relapse-remitting and chronic-progressive forms of multiple sclerosis in mouse models. Sci Rep. 2019;9:6923.
61. Castillo-Gómez E, Oliveira B, Tapken D, Bertrand S, Klein-Schmidt C, Pan H, et al. All naturally occurring autoantibodies against the NMDA receptor subunit NR1 have pathogenic potential irrespective of epitope and immunoglobulin class. Mol Psychiatry. 2017;22:1776-84.

62. Zhang J, Jacobi AM, Wang T, Berlin R, Volpe BT, Diamond B. Polyreactive autoantibodies in systemic lupus erythematosus have pathogenic potential. J Autoimmun. 2009;33:270-4.

\section{ACKNOWLEDGEMENTS}

This study was supported by the Max Planck Society, the Deutsche Forschungsgemeinschaft (DFG, German Research Foundation) TRR 274/1 2020-408885537. KAN is funded by Adelson Medical Research Foundation and an ERC Advanced Grant.

\section{AUTHOR CONTRIBUTIONS}

Concept, design, and supervision of the study: $\mathrm{HE}$; data acquisition/analysis/ interpretation: JBHW, MH, SAB, SZ, AR, SA, NB, AAS, SR, MH, WS, KAN, FL, and HE; drafting the paper: HE together with JBHW; Drafting display items: JBHW, SZ, together with $\mathrm{HE}$; All authors read and approved the final version of the paper.

\section{FUNDING}

Open Access funding enabled and organized by Projekt DEAL.

\section{COMPETING INTERESTS}

WS is a member of the board and holds stocks in Euroimmun AG. All other authors declare no competing financial or other interests.

\section{ADDITIONAL INFORMATION}

Correspondence and requests for materials should be addressed to H.E.

Reprints and permission information is available at http://www.nature.com/ reprints

Publisher's note Springer Nature remains neutral with regard to jurisdictional claims in published maps and institutional affiliations.

\begin{abstract}
Open Access This article is licensed under a Creative Commons Attribution 4.0 International License, which permits use, sharing, adaptation, distribution and reproduction in any medium or format, as long as you give appropriate credit to the original author(s) and the source, provide a link to the Creative Commons license, and indicate if changes were made. The images or other third party material in this article are included in the article's Creative Commons license, unless indicated otherwise in a credit line to the material. If material is not included in the article's Creative Commons license and your intended use is not permitted by statutory regulation or exceeds the permitted use, you will need to obtain permission directly from the copyright holder. To view a copy of this license, visit http://creativecommons. org/licenses/by/4.0/.
\end{abstract}

(c) The Author(s) 2021 